\title{
Bergamot natural products eradicate cancer stem cells (CSCs) by targeting mevalonate, Rho-GDI-signalling and mitochondrial metabolism
}

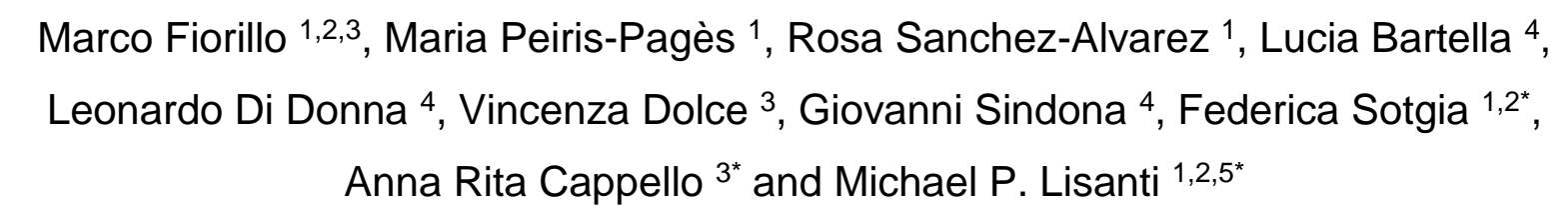




\section{Abstract}

Here, we show that a 2:1 mixture of Brutieridin and Melitidin, termed "BMF", has a statin-like properties, which blocks the action of the rate-limiting enzyme for mevalonate biosynthesis, namely HMGR (3-hydroxy-3-methylglutaryl-CoAreductase). Moreover, our results indicate that BMF functionally inhibits several key characteristics of CSCs. More specifically, BMF effectively i) reduced ALDH activity, ii) blocked mammosphere formation and iii) inhibited the activation of CSC-associated signalling pathways (STAT1/3, Notch and Wnt/beta-catenin) targeting Rho-GDIsignaling. In addition, BMF metabolically inhibited mitochondrial respiration (OXPHOS) and fatty acid oxidation (FAO). Importantly, BMF did not show the same toxic side-effects in normal fibroblasts that were observed with statins. Lastly, we show that high expression of the mRNA species encoding HMGR is associated with poor clinical outcome in breast cancer patients, providing a potential companion diagnostic for BMF-directed personalized therapy.

\section{Keywords}

CSCs signalling, HMGR, Bergamot, mevalonate pathway inhibitor, Rho-GDIsignalling, breast cancer.

\section{Introduction}

Clinical data and epidemiological studies both support the idea that cholesterollowering drugs are able to reduce cancer incidence and cancer related mortality [13], by decreasing cholesterol (either locally synthesised or circulating levels) [4, 5]. Moreover, multiple studies have shown the anti-proliferative effects of statins against both cancer cells and cancer stem cells (CSCs) [6, 7]. CSCs represent a distinct subpopulation of cancer cells, with high tumorigenicity [8], that are able to regenerate the tumor by self-renewal and by the generation of new progenitor cells [9, 10]. CSCs are a small percentage of the total cancer cell population, but are responsible for patient relapse, metastasis and for their particular ability [11] to resist and survive conventional chemotherapy and radiation [12, 13]. Existing cancer treatments are usually unable to eradicate CSCs. Indeed new drugs are currently being developed that are focused on targeting CSC signalling pathways, self-renewal and metastasis. 
These new therapies would be used in conjunction with more conventional cancer therapies $[14,15]$.

Recently, statins have been proposed as new drugs to defeat CSCs, via mevalonate pathway inhibition [16]. Previous studies have shown that the modulation of this metabolic pathway is a key factor for breast CSC maintenance [17]. Statins are strong competitive inhibitors of 3-hydroxy-3-methylglutaryl-CoA-reductase enzyme (HMGR), an enzyme which catalyzes the rate-limiting step in mevalonate biosynthesis and regulates isoprene formation. Numerous small G-proteins depend on prenylation, which is regulated by isoprenes. Thus, G-protein signalling pathways are regulated by statins, through the reversible inhibition of the prenylation process $[18,19]$. Despite the fact that statins are currently considered safe, many patients are statin-intolerant and show significant side-effects during their treatment, in combination with common anticancer drugs, highlighting the urgency of finding new drugs acting like statins [20,21]. Some foods possess several statin-like therapeutic properties [22], possibly due to the presence of flavonoids, pectins and ascorbic acid, which have a high antioxidant potential [23], and may interfere with cholesterol and isoprene metabolism [24]. Their intake is associated with a reduced risk of numerous chronic diseases, such as cancerous processes [25]. Flavonoids also exhibit antiviral, anti-microbial, and anti-inflammatory activities [26-28], and support a strong immune response [29]. In relation to that, the Bergamot fruit (Citrus bergamia Risso) has attracted attention for its remarkable flavonoid composition [30-32]. Recently, we detected two flavonoids (extracted from the Bergamot fruit), containing a 3-hydroxy3-methylglutaric acid (HMG) moiety, called Brutieridin and Melitidin [33]. Their inhibitory potential against the HMGR enzyme was previously substantiated in vitro and their hypo-cholesterolemic effects were also verified in vivo [34]. In the present study, Brutieridin and Melitidin were purified from the fruit of the Bergamot tree $(\sim 99 \%)$ and mixed together in an enriched flavonoid fraction (termed "BMF"), corresponding to $70 \%$ Brutieridin and $30 \%$ Melitidin. By comparing the proteomic profiles of 3D-spheroids to cancer cells grown as monolayers, we identified the overexpression of enzymes involved in the mevalonate pathway in CSCs. This finding prompted us to investigate the therapeutic potential of BMF to target CSCs propagation, via HMGR blockade. For this purpose, we compared BMF to the activity of common FDA-approved statins (Pravastatin and Simvastatin). Indeed, we found 
that BMF inhibits several characteristics of CSC behaviour, including mammosphere formation [35], ALDH content [36], mitochondrial respiration and fatty acid oxidation [37], as well as several stemness-related signalling pathways [38], such as the STAT1/3, Notch and Wnt/beta-catenin pathways, in MCF7 breast cancer cells. On the contrary, BMF does not show that same cytotoxic side-effects on normal human fibroblasts, that we observed with Pravastatin and Simvastatin. Furthermore, the addition of mevalonate to the culture media of MCF7 cells was able to effectively restore their ability to grow in suspension, as well as rescue their ALDH content. Thus, BMF may be a more effective, non-toxic, all-natural, therapeutic for the eradication of CSCs, via mevalonate pathway inhibition.

\section{Material and Methods}

\subsection{Experimental Model and Subject Details}

Human breast cancer cell lines (T47D and MCF7) were obtained commercially from the ATCC. hTERT-BJ1 cells are human foreskin fibroblasts, that were originally obtained from Clontech, Inc. All cell lines were maintained in Dulbecco's Modified Eagle Medium (DMEM; GIBCO) supplemented with 10\% FBS, 1\% Glutamax and 1\% Penicillin-Streptomycin. All cell lines were maintained at $37^{\circ} \mathrm{C}$ in $5 \% \mathrm{CO} 2$. MCF7 cells were used for lentiviral transfection.

\subsection{Preparation of Brutieridin and Melitidin (BMF)}

Bergamot fruit was collected in December 2012 (in Calabria, Italy) and then stored at $-20^{\circ} \mathrm{C}$. Briefly, $7 \mathrm{~kg}$ of fruits were squeezed to obtain the juice $(2000 \mathrm{~mL})$ which was filtered and passed through a $10 \mathrm{~g} \mathrm{C}_{18}$ cartridge (Supelco, USA) in $50 \mathrm{~mL}$ aliquots. The loaded stationary phase was initially washed with water $(2 \times 50 \mathrm{~mL})$ to remove the sugars and water soluble fraction, and then eluted with $50 \mathrm{~mL}$ of methanol to collect the flavonoid fraction. Each aliquot passed through the resin provided ca. $80 \mathrm{mg}$ raw flavonoid fraction, for a total amount of $3.2 \mathrm{~g}$. The polyphenolic fraction coming from the SPE step was loaded onto a glass column $(46 \times 2.6 \mathrm{~cm})$ from Buchi (USA) packed with $100 \mathrm{~g}$ of $\mathrm{C}_{18}$ 80-60 mesh (Sigma-Aldrich, USA) and connected to a Perkin Elmer 200 LC binary pump. $\mathrm{H}_{2} \mathrm{O}$ (solvent $\mathrm{A}$ ) and $\mathrm{CH}_{3} \mathrm{OH}$ (solvent $\mathrm{B}$ ) at the flow rate of $1.5 \mathrm{~mL} / \mathrm{min}$ were used as elution solvents at the following gradient steps: isocratic at $100 \%$ A for 40 min.; linear gradient from $100 \%$ A to $70 \%$ A in 60 min.; 
isocratic at $70 \%$ A for 60 min.; linear gradient from $70 \%$ A to $40 \%$ A in $60 \mathrm{~min}$.; isocratic at $40 \%$ A for 60 min.; linear gradient from $40 \% A$ to $0 \% A$ in 10 min.; washing of the column at $0 \% \mathrm{~A}$ for $60 \mathrm{~min}$. The initial water elution was discarded and the collected fractions starting from min 40 (20 mL each) were monitored by HPLC/UVMS using a Fractionlynx semi-preparative HPLC system (Waters Corp., Milford, MA, USA). The system was composed of an autosampler/collector Waters 2767 Sample Manager, a 600E pump working in analytical mode, a $486 \mathrm{~nm}$ UV detector and a ZMD mass spectrometer equipped with an ESI source working in negative ionization mode. The HPLC separation was achieved using a $250 \times 4.6 \mathrm{~mm}, 5 \mu \mathrm{m}$ reversed phase $\mathrm{C}_{18}$ Luna-Phenomenex column at a flow rate of $1 \mathrm{~mL} / \mathrm{min}$. The run time was $70 \mathrm{~min}$ and the mobile phase was composed by $0.1 \%$ formic acid in water (solvent $A$ ) and methanol (solvent $B$ ). The chromatographic run $(70 \mathrm{~min})$ consisted of the following steps: isocratic at $80 \%$ A for 7 min; linear gradient from $80 \%$ A to $40 \%$ A in $33 \mathrm{~min}$; isocratic at $40 \%$ A for $5 \mathrm{~min}$; linear gradient from $40 \% \mathrm{~A}$ to $20 \% \mathrm{~A}$ in $5 \mathrm{~min}$; isocratic at $20 \% \mathrm{~A}$ for $7 \mathrm{~min}$; linear gradient from $20 \% \mathrm{~A}$ to $80 \% \mathrm{~A}$ in $5 \mathrm{~min}$; equilibration of the column for $8 \mathrm{~min}$. The UV detector was set at $280 \mathrm{~nm}$. The MS conditions were the following: capillary voltage $-3.15 \mathrm{kV}$, cone voltage $-3 \mathrm{~V}$, extractor $-2 \mathrm{~V}$, RF lens -0.34 $\mathrm{V}$, source block and desolvation temperature $120,250{ }^{\circ} \mathrm{C}$ respectively, ion energy $0.5 \mathrm{~V}, \mathrm{LM}$ resolution 14.5, HM resolution 15.0 and multiplier $650 \mathrm{~V}$. The nebuliser gas was set to $650 \mathrm{~L} / \mathrm{h}$. The fractions coming from the separation and containing respectively compound, brutieridin and melitidin, were evaporated under reduced pressure, lyophilized and submitted to the purification step, using the Fractionlynx system working in semi-preparative mode, at the same experimental condition

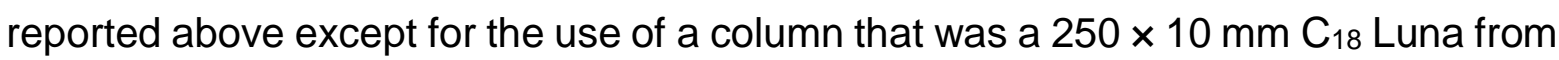
Phenomenex (Torrance, CA) and for a chromatographic run (30 min; isocratic at $55 \%$ A). The flow rate was set to $4.7 \mathrm{~mL} / \mathrm{min}$, and the fractions were collected every 30 seconds, while the injected sample volume was $1 \mathrm{~mL}$. The purity of HMG flavonoid was verified by HPLC/UV.

\subsection{Lentiviral transduction}

Lentiviral plasmids, packaging cells and reagents were purchased from Genecopoeia. Forty-eight hours after seeding, 293Ta packaging cells were transfected with lentiviral vectors encoding HMGR or the empty vector alone (EXNEG-Lv105), using Lenti-PacTM HIV Expression Packaging Kit, according to the 
manufacturer's instructions. Two days post-transfection, lentivirus-containing culture medium was passed through a $0.45 \mu \mathrm{m}$ filter and added to the target cells (MCF7 cells), in the presence of $5 \mu \mathrm{g} / \mathrm{ml}$ Polybrene. Infected cells were selected with a concentration of $1.5 \mu \mathrm{g} / \mathrm{ml}$ of puromycin.

\subsection{Sulfo-rhodamine $B$ (SRB) assay}

SRB measures total biomass by staining cellular proteins [39]. After $48 \mathrm{~h}$ treatment, cells were fixed in $10 \%$ trichloroacetic acid (T9159, Sigma) for $1 \mathrm{~h}$ at $4^{\circ} \mathrm{C}$, stained with SRB (S9012, Sigma) for 15 minutes, and washed 3 times with $1 \%$ acetic acid (27225, Sigma). The incorporated dye was solubilized with $10 \mathrm{mM}$ Tris-HCl, pH 8.8 (T1503, Sigma). Absorbance was spectrophotometrically measured at $540 \mathrm{~nm}$ in a FluoStar Omega plate reader (BMG Labtech). Background measurements were subtracted from all values.

\subsection{Cell cycle analysis}

Control and drug-treated MCF7 cells were subjected to cell-cycle analysis by FACS [40]. Briefly, MCF7 cells were treated with $100 \mu \mathrm{M}$ and $1 \mathrm{mM}$ BMF or Pravastatin. After 72 hours, the cells were harvested and their nuclei stained with DAPI. $1 \times 10^{6}$ cells, for each condition, were fixed with cold ethanol (70\%) for $1 \mathrm{~h}$ on ice, centrifuged, and washed twice in cold PBS. The samples were then incubated with RNase A (20 $\mu \mathrm{g} / \mathrm{ml}$ ) and stained with Propidium lodide (Pl; $100 \mu \mathrm{g} / \mathrm{ml}$ ) (Sigma-Aldrich). Following a $30 \mathrm{~min}$ incubation at $37^{\circ} \mathrm{C}$, the cells were analysed $(50,000$ events per condition) using FACS (BD Fortessa). Gated cells were manually categorised into cell-cycle stages.

\subsection{Seahorse XFe96 metabolic flux analysis.}

Real-time oxygen consumption rates (OCR), extracellular acidification rates (ECAR) and fatty acid oxidation (FAO) rates for MCF7 cells and normal fibroblasts (hTERTBJ1 cells) treated with BMF, pravastatin and simvastatin were determined using the Seahorse Extracellular Flux (XFe96) analyzer (Seahorse Bioscience, USA). Briefly, $1 \times 10^{4}$ cells per well were seeded into XFe96 well cell culture plates, and incubated overnight to allow cell attachment. Then, cells were treated with BMF, pravastatin and simvastatin $(100 \mu \mathrm{M}$ and $1 \mathrm{mM})$ for 72 hours. Vehicle alone (DMSO) control cells were processed in parallel. After 72 hours of incubation, cells were washed in pre-warmed 
XF assay media (or for OCR measurement, XF assay media supplemented with $10 \mathrm{mM}$ glucose, $1 \mathrm{mM}$ Pyruvate, $2 \mathrm{mM}$ L-glutamine and adjusted at $7.4 \mathrm{pH}$ ). Cells were then maintained in $175 \mu \mathrm{L} /$ well of $\mathrm{XF}$ assay media at $37^{\circ} \mathrm{C}$, in a non- $\mathrm{CO}_{2}$ incubator for 1 hour. During the incubation time, we loaded $25 \mu \mathrm{L}$ of $80 \mathrm{mM}$ glucose, $9 \mu \mathrm{M}$

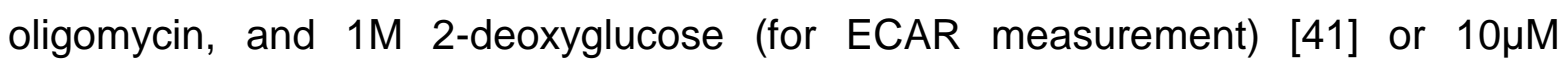
oligomycin, $9 \mu \mathrm{M} \mathrm{FCCP}, 10 \mu \mathrm{M}$ rotenone, $10 \mu \mathrm{M}$ antimycin $\mathrm{A}$ (for OCR measurement) $[42,43]$, in XF assay media into the injection ports in the XFe96 sensor cartridge. The fatty acid oxidation (FAO) was evaluated using an XF assay for oxidation of exogenous and endogenous FAs. Similarly, $1.5 \times 10^{3}$ cells were seeded in XF Cell Culture Microplates and allowed to grow overnight in typical growth medium. The growth medium was then replaced (after 24h) with substrate-limited medium contained BMF (1 mM), glucose $(0.5 \mathrm{mM})$, GlutaMAX $(1 \mathrm{mM})$, carnitine $(0.5 \mathrm{mM})$ and $1 \%$ FBS to deplete endogenous substrates within the cell (glycogen, triglycerides, amino acids), thus priming the cells to oxidize exogenous FAs. Carnitine was added fresh the day of the media change and serum to deplete endogenous substrates within the cell (glycogen, triglycerides, amino acids), thus priming the cells to oxidize exogenous FAs. Prior to the assay ( 45 minutes before) the cells were washed twice times with $\mathrm{FAO}$ Assay Medium contained $\mathrm{NaCl}(111 \mathrm{mM}), \mathrm{KCl}(4.7 \mathrm{mM}), \mathrm{CaCl}_{2}(1.25$ $\mathrm{mM}), \mathrm{MgSO}_{4}(2 \mathrm{mM}), \mathrm{NaH}_{2} \mathrm{PO}_{4}(1.2 \mathrm{mM})$, supplemented with glucose $(2.5 \mathrm{mM})$, carnitine $(0.5 \mathrm{mM})$, and HEPES $(5 \mathrm{mM})$ on the day of the assay, adjusted to $\mathrm{pH} 7.4$ at $37^{\circ} \mathrm{C}$. The $\mathrm{FAO}$ assay medium was added to the plate $(135 \mu \mathrm{L} /$ well $)$ and incubated in a non-CO2 incubator for 30 minutes at $37^{\circ} \mathrm{C}$. The cartridge was loaded following the OCR protocol, as described before. After 30 minutes $10 \mathrm{mM}$ stock solution of Etomoxir (Eto) was diluted to $400 \mu \mathrm{M}$ in FAO Assay Medium and was added $15 \mu \mathrm{L}$ to the appropriate wells. The final concentration of Eto in the wells was $40 \mu \mathrm{M}$. The plate was incubated for 15 minutes at $37^{\circ} \mathrm{C}$ in a non-CO2 incubator. Just prior to starting the assay, $30 \mu \mathrm{L}$ of XF Palmitate-BSA FAO Substrate or BSA was added to the appropriate wells and immediately the XF Cell Culture Microplate was inserted into the XFe96 Analyzer and the XF Cell Mito Stress Test was run with the command protocol. Measurements were normalized by protein content (SRB and Bradford assay). Data sets were analyzed using XFe96 software and GraphPad Prism software, using one-way ANOVA and Student's t-test calculations. All experiments were performed in quintuplicate, three times independently. 


\subsection{Quantitative assessment of CSC signalling pathways}

The Cignal Lenti reporter assay (luc) system (Qiagen) was chosen for monitoring the activity of several signal transduction pathways in MCF7 cells [44]. The responsive luciferase constructs encode the firefly luciferase reporter gene under the control of a minimal $(\mathrm{m}) \mathrm{CMV}$ promoter and tandem repeats of response elements for each pathway. The following constructs were used: TCF/LEF(luc) for Wnt signal transduction (CLS-018L); STAT3(luc) for transcriptional activity of STAT3 (CLS6028L); RBP-Jk(luc) for Notch-induced signaling (CLS-014L); ARE(luc) for Nrf2- and Nrf1-mediated antioxidant responses (CLS-2020L); GAS(luc) for Interferon gammainduced Stat1-signal transduction (CLS-009L); and SMAD(luc) for TGFß-induced signal transduction (CLS-017L). Briefly, 1 × $10^{5}$ MCF7 cells were seeded in 12-well plates. Once cells were attached, the viral particles were diluted 1:10 in complete culture media containing polybrene (sc-134220, Santa Cruz), and added to the cells. Puromycin treatment (P9620, Sigma) was started 48 hours later, in order to select stably infected cells.

\subsection{Luciferase assays}

The Luciferase Assay System (E1501, Promega Kit) was used on all luciferase reporter MCF7 cells treated with BMF. Briefly, $6 \times 10^{3}$ MCF7 cells were seeded in black-walled 96-well plates and then were treated with BMF $1 \mathrm{mM}$. As controls, vehicle-alone treated cells were run in parallel. Six replicates were used for each condition. After 72 hours of treatment, luciferase assays were performed according to the manufacturer's instructions. Light signal was acquired for 2 minutes in photons/second in the Xenogen VivoVision IVIS Lumina (Caliper Life Sciences), and the results were analysed using Living Image 3.2 software (Caliper Life Sciences). Luminescence was normalized using SRB (to determine total cellular protein), as a measure of MCF7 cell viability.

\subsection{GM-CSF and IL-8 ELISA assays}

To evaluate the potential anti-inflammatory effects of BMF, we utilized the IL-8 Human SimpleStep (Ab 174442, Abcam) and the GM-CSF Human SimpleStep (Ab 174448, Abcam) ELISA kits. The experiments were performed on pre- collect cellular media, after $72 \mathrm{~h}$ of treatment with BMF and pravastatin, in MCF7 cells. The ELISA plates was pre-warmed a $25^{\circ}$ for 30 minutes before use. Afterwards, $50 \mu$ of media 
and $50 \mu$ of cocktail antibody were added in each well and left at $25^{\circ} \mathrm{C}$ for $1 \mathrm{~h}$ mixing at $400 \mathrm{rpm}$. After 1 hour, each well was washed three times with $350 \mu \mathrm{l}$ of wash buffer and $100 \mu \mathrm{l}$ of TMB substrate were added in each well. The plates were incubated in a dark room for 10 minutes mixing at $400 \mathrm{rpm}$. After 10 minutes, we added $100 \mu \mathrm{l}$ of stop solution and the plate was incubated for 1 minute. Lastly, the plate was read using a FLUOstar Omega Microplate Reader at $600 \mathrm{~nm}$.

\subsection{MCF7 3D-mammosphere formation}

A single cell suspension was prepared using enzymatic (1x Trypsin-EDTA, Sigma Aldrich, \#T3924), and manual disaggregation (25 gauge needle), to create a single cell suspension. Cells were plated at a density of $500 \mathrm{cells} / \mathrm{cm}^{2}$ in mammosphere medium (DMEM-F12 + B27 + $20 \mathrm{ng} / \mathrm{ml}$ EGF + PenStrep) under non-adherent conditions, in culture dishes pre-coated with (2-hydroxyethylmethacrylate) (polyHEMA, Sigma, \#P3932), called "mammosphere plates" [45]. Then, the cells were pretreated for 72 hours with BMF $(100 \mu \mathrm{M}$ and $1 \mathrm{mM})$ and Pravastatin $(100 \mu \mathrm{M}$ and 1 $\mathrm{mM}$ ). Afterwards, they were trypsined and seeded in mammosphere plates or treated directly in mammosphere plates with BMF $(100 \mu \mathrm{M}$ and $1 \mathrm{mM})$ and Pravastatin (100 $\mu \mathrm{M}$ and $1 \mathrm{mM}$ ); this was carried out in presence or absence of mevalonate $1 \mathrm{mM}$ and cholesterol $10 \mu \mathrm{M}$. Vehicle alone (DMSO) control cells were processed in parallel. Cells were grown for 5 days and maintained in a humidified incubator at $37^{\circ} \mathrm{C}$. After 5 days of culture, 3D-spheres $>50 \mu \mathrm{m}$ were counted using an eye piece ("graticule"), and the percentage of cells plated which formed spheres was calculated and is referred to as percent mammosphere formation, and was normalized to one ( $1=$ $100 \%$ MSF).

\subsection{ALDEFLUOR assay}

ALDH activity was assessed in MCF7cells. The ALDEFLUOR kit (StemCell technologies, Durham, NC, USA) was used to isolate the population with high ALDH enzymatic activity by FACS (Fortessa, BD Bioscence). Briefly, $1 \times 10^{5}$ were incubated in $1 \mathrm{ml}$ ALDEFLUOR assay buffer containing ALDH substrate $(5 \mu \mathrm{l} / \mathrm{ml})$ for 40 minutes at $37^{\circ} \mathrm{C}$. In each experiment a sample of cells was stained under identical conditions with $30 \mathrm{mM}$ of diethylaminobenzaldehyde (DEAB), a specific ALDH inhibitor, as a negative control The ALDH-positive population was established, 
according to the manufacturer's instructions and was evaluated using 20.000 cells. An ALDEFLUOR-positive signal was detected in cell lines treated with BMF $(100 \mu \mathrm{M}$ and $1 \mathrm{mM})$ and/or Pravastatin ( $100 \mu \mathrm{M}$ and $1 \mathrm{mM})$, as compared with controls.

\subsection{Label-free unbiased semi-quantitative proteomics analysis}

Cell lysates were prepared for trypsin digestion by sequential reduction of disulphide bonds with TCEP and alkylation with MMTS. Then, the peptides were extracted and prepared for LC-MS/MS. All LC-MS/MS analyses were performed on an LTQ Orbitrap $\mathrm{XL}$ mass spectrometer (Thermo Scientific, San Jose, CA) coupled to an Ultimate 3000 RSLCnano system (Thermo Scientific, formerly Dionex, The Netherlands). Xcalibur raw data files acquired on the LTQ-Orbitrap XL were directly imported into Progenesis LCMS software (Waters Corp., Milford, MA, formerly Non-linear dynamics, Newcastle upon Tyne, UK) for peak detection and alignment [46]. Data were analyzed using the Mascot search. Five technical replicates were analyzed for each sample type.

\subsection{Ingenuity pathway analysis (IPA)}

Unbiased interrogation and analysis of our proteomic data sets was carried out by employing a bioinformatics platform, known as Ingenuity Pathway Analysis (IPA) (Ingenuity systems, http://www.ingenuity.com). IPA assists with data interpretation, via the grouping of differentially expressed genes or proteins into known functions and pathways. Functional protein networks and upstream regulator analysis with differently expressed proteins were presented, along with a Z-score. Pathways with a $z$ score of $>+2$ were considered as significantly activated, while pathways with a $z$ score of $<-2$ were considered as significantly inhibited. For a more detailed explanation regarding Z-scores, please see: Ingenuity systems, http://www.ingenuity.com.

\subsection{Western blotting}

Cells were lysed in buffer ( $1 \%(\mathrm{v} / \mathrm{v})$ Triton X-100, 50mM HEPES, pH 7, 1 mM EDTA, $1 \mathrm{mM}$ EGTA, $150 \mathrm{mM} \mathrm{NaCl}, 100 \mathrm{mM}$ sodium fluoride, $1 \mathrm{mM} \mathrm{Na3VO}$, and one tablet of Complete TM inhibitor mix (Roche Applied Science, Indianapolis) per $25 \mathrm{~mL}$ of buffer and loaded on to SDS-polyacrylamide gels. Blots were incubated with the respective primary antibodies diluted in tris-buffered saline and tween 20 (TBST) 
339 (containing $0.1 \%$ Tween20 and $5 \%$ milk powder) and incubated overnight at $4{ }^{\circ} \mathrm{C}$.

340 Then, blots were washed and incu-bated with appropriate secondary antibodies (GE Healthcare) and detected using SuperSignal West Pico Chemiluminescent Substrate (Pierce, Rockford, IL). Antibodies and their dilutions used for Western blot analysis were as follows: rabbit anti-HMGCR (Santa-Cruz; 1:500), mouse anti-ERa (6F11, Novocastra; 1:1,000), rabbit anti-p27Kip1 (Dako; 1:500), rabbit anti-cyclinD (Cell signalling; 1:1,000), rabbit anti-cyclinE (Cell signalling, 1:1,000), mouse anti-p53 (Sigma-Aldrich; 1:500), mouse anti-Rb (Santa-Cruz; 1:500), mouse total OXPHOS anti-human cocktail (Abcam; 1:1,000), anti- $\beta$-tubulin (Sigma-Aldrich; 1:5,000), anti- $\beta$ actin (Sigma-Aldrich; 1:10,000).

\subsection{Kaplan-Meier}

All graphs (see Figure 7) were plotted using microarray data from human breast cancer patients, determined using an online survival analysis tool. Kaplan-Meier correlations are plotted for high (above median, in Red) and low (below median, in Black) gene expression. Biased array data were excluded from the analysis. Hazardratios were calculated, at the best auto-selected cut-off, and p-values were calculated using the logrank test and plotted in R. K-M curves were also generated online using the K-M-plotter (as high-resolution TIFF files), using univariate analysis: http://kmplot.com/analysis/index.php?p = service\&cancer = breast. This allowed us to directly perform in silico validation of HMGR as a potential biomarker. The most updated version of the database (2017) was utilized, for all these analyses.

\subsection{Quantification and Statistical Analysis}

All analyses were performed with GraphPad Prism 6. Data were presented as mean \pm SEM ( \pm SD where indicated). All experiments were conducted at least three times, with $\geq 3$ technical replicates per experiment, unless otherwise stated with representative data shown. Statistically significant differences were determined using the Student's t test or the analysis of variance (ANOVA) test. For the comparison among multiple groups, one-way ANOVA were used to determine statistical significance. $P \leq 0.05$ was considered significant and all statistical tests were twosided.

\subsection{Contact for Reagent and Resource}


372 Further information and requests for resources and reagents should be directed to

373 and will be fulfilled by the Lead Contact, Michael P. Lisanti

374 (michaelp.lisanti@gmail.com)

\section{Supplemental Information}

377 Supplemental Information includes two figures and two tables.

\section{Results}

\subsection{MCF7 and T47D mammospheres show the over-expression of key enzymes} involved in mevalonate metabolism, including HMGR, as revealed by proteomics analysis

MCF7 cells, grown either as i) a monolayer or ii) as 3D-mammospheres in suspension, were subjected to unbiased label-free proteomics analysis. This strategic approach would allow us to identify which proteins are specifically up-regulated or down-regulated, during mammosphere suspension cultures. For comparison purposes, we also performed the same type of analysis (monolayer vs. suspension culture) on a second independent ER (+) breast cancer cell line, namely T47D cells. The differential expression patterns of proteins in these four data sets was then subjected to Ingenuity Pathway Analysis (IPA), to determine possible alterations in canonical signaling pathways (Figure S1A) and toxicity functions (Figure S1B). Importantly, this comparative analysis showed that these two independent cell lines behaved similarly, in a conserved fashion. For simplicity, we focused on the proteins involved in cholesterol biosynthesis (the mevalonate pathway); note that this pathway is significantly up-regulated in mammospheres, as compared to monolayer cell cultures (Figure S1B) $(p<0.05)$. These results are summarized in Figure S2A and S2B. Remarkably, 25 proteins involved in the mevalonate pathway and cholesterol biosynthesis, were found to be up-regulated in MCF7 mammospheres, as compared to MCF7 monolayer cells. Moreover, 22 proteins were found to be up-regulated in T47D mammospheres, as compared to T47D monolayer cells. This represents an overlap of $88 \%$ (22 out of 25), as shown in the Venn diagram presented in Figure 2SA. Therefore, we conclude that cholesterol biosynthesis appears to be highlyactivated or enhanced in cancer cells grown in suspension cultures. As these 3Dcultures are thought to be enriched in CSCs and progenitor cells, cholesterol 
biosynthesis may be a key biosynthetic pathway that is necessary or required for maintaining "stemness" in cancer cells (Figure 2SB). As a consequence of these findings, we hypothesised that an inhibitor of 3-hydroxy-3-methylglutaryl-CoA reductase (HMGR), a key enzyme in mevalonate metabolism, would effectively inhibit the survival and propagation of breast CSCs. This prompted us to test the effects of BMF on cancer cell proliferation and CSC propagation.

\subsection{BMF inhibits the enzymatic activity of HMGR}

In the present study, two novel molecules we previously isolated and identified (ref. Didonna et al. 2009), were purified as HMG conjugates of Neohesperidin and Naringin, namely: i) Brutieridin [hesperetin 7-(2"-R-rhamnosyl-6"-(3"'--hydroxy-3"'"methylglutaryl)-glucoside] and ii) Melitidin [naringenin 7-(2"-R-rhamnosyl-6"-(3"'-' hydroxy-3"'"-methylglutaryl)-glucoside] (Figure 1B). Several analytical experiments were performed to confirm and validate their structures (Figure 1B); in particular, we used UV, IR spectra and HPLC-MS/UV (Figure 1C). We find that Brutieridin and Melitidin are present in the Bergamot fruit in a concentration range of $\sim 300-500$ ppm and $150-300 \mathrm{ppm}$, respectively, as a function of the ripening stage; these compounds may be found either in the juice or in the albedo and flavedo of the Bergamot fruit skin. The "signature moiety" of Brutieridin and Melitidin is the presence of a 3-hydroxy3-methyl glutaryl (HMG) moiety, esterified on the nehoesperidose (sugar) moiety (Figure 1B). Therefore, we predicted that they would exhibit an inhibitory effect against HMGR (3-hydroxy-3-methylglutaryl-CoA reductase), thereby reducing its enzymatic activity. This hypothesis was confirmed using a well-established HMGR activity assay (Figure 1D). The assay is based on the spectrophotometric measurement of a decrease in absorbance at $340 \mathrm{~nm}$, which represents the oxidation of NADPH by the catalytic subunit of HMGR, in the presence of the substrate HMGCoA. Different concentrations of Brutieridin, Melitidin and BMF were evaluated to determine the optimal inhibitory concentrations for blocking HMGR activity (not shown). Brutieridin and Melitidin decreased HMGR activity by $55 \%$ and $65 \%$, respectively, at $100 \mu \mathrm{M}$. This result confirms that Brutieridin and Melitidin have a statin-like inhibitory effect on HMGR activity. However, greater inhibition capacity, of $\sim 85 \%$, was detected when the BMF fraction, containing both molecules, was analyzed (Figure 1D), as compared to when both molecules were analyzed individually. This is indicative of an additive effect. Thus, in the present work, we chose 
to investigate the effects of BMF, as an HMG-flavanone fraction (defined as a purified $\sim 2: 1$ mixture, composed of $70 \%$ Brutieridin and 30\% Melitidin).

\subsection{BMF reduces MCF7 and MCF7-HMGR cell growth}

The effects of BMF on cell proliferation were first examined using MCF7 cells and compared with two commercial inhibitors of mevalonate biosynthesis: Pravastatin and Simvastatin. Importantly, MCF7 breast cancer cells endogenously express HMGR. However, in parallel, we also generated an MCF7 cell line over-expressing recombinant HMGR, via lenti-viral transduction. Over-expression of HMGR in MCF7HMGR cells was indeed confirmed by Western blot analysis, as compared with MCF7 cells transduced with the empty vector alone (Lv-105) (Figure 2A). The cells were treated, for 72 or 120 hours, with either BMF (100 $\mu \mathrm{M}$ and $1 \mathrm{mM}$; Figure 2B upper panel), Pravastatin (100 $\mu \mathrm{M}$ and $1 \mathrm{mM}$; Figure 2B middle panel), or Simvastatin (10, 50, $100 \mu \mathrm{M}$ and $1 \mathrm{mM}$; Figure 2B lower panel). Note that Figure 2B shows a significant dose-dependent reduction in cell proliferation in MCF7-HMGR cells, as compared with MCF7 cells. The observed IC 50 value was between $100 \mu \mathrm{M}$ and $1 \mathrm{mM}$ for BMF and Pravastatin and was approximately $10 \mu \mathrm{M}$ for Simvastatin, in both cell lines. Likewise, the toxicity of BMF, Pravastatin and Simvastatin was also examined in a normal fibroblast cell line (hTERT-BJ1). Figure 2B shows that Pravastatin and Simvastatin are toxic for hTERT-BJ1 cells (IC50 values ranging between $10 \mu \mathrm{M}$ and $50 \mu \mathrm{M})$. Surprisingly, BMF did not exhibit any toxic effects with hTERT-BJ1 cells, after $72 \mathrm{~h}$ and $120 \mathrm{~h}$ of treatment.

\subsection{BMF arrests $M C F 7$ cells in $G_{0} / G_{1}$ phase of the cell cycle}

To evaluate the underlying mechanism(s) of growth inhibition by BMF, cell cycle profiles were analysed, using MCF7 cells, after $72 \mathrm{~h}$ of treatment with BMF and Pravastatin (100 $\mu \mathrm{M}$ and $1 \mathrm{mM}$ each) (Figure 2C). All treatments resulted in $\mathrm{G}_{0} / \mathrm{G}_{1}$ cell cycle arrest and reduced S phase, in a dose-dependent manner, as compared to vehicle-alone controls.

\subsection{BMF decreases mitochondrial respiration, by reducing OXPHOS and} exogenous fatty acid oxidation (FAO) in MCF7 cells

The metabolic phenotype of MCF7-HMGR cells was assessed using the Seahorse 
472 XFe96 metabolic flux analyser; MCF7-EV (empty vector control) cells were also analyzed in parallel, as a negative control. Both isogenic cell lines were subjected to glycolytic and mitochondrial stress tests (Figure 3A and 3B). Notably, no differences were observed in extracellular acidification rates (ECAR) (Figure 3A), while the oxygen consumption rate (OCR) showed a significant increase, but only in MCF7HMGR cells, as compared to MCF7-EV cells. Therefore, over-expression of HMGR "boosts" mitochondrial metabolism, through the production of mevalonate. Next, to evaluate if BMF inhibits mitochondrial function in cancer cells, OCR was assessed in parental MCF7 cell monolayers, treated for 72 hours with BMF, Pravastatin or Simvastatin (each at $100 \mu \mathrm{M}$ and $1 \mathrm{mM}$ ) (Figure 3C). As predicted, our results show that BMF treatment effectively decreases mitochondrial respiration in MCF7 cells. Significant reductions in OCR were observed in MCF7 cells treated with BMF (1 mM). Similarly, after 72 hour, Pravastatin $(100 \mu \mathrm{M}$ and $1 \mathrm{mM})$ and Simvastatin (100 $\mu \mathrm{M}$ and $1 \mathrm{mM}$ ) both showed greatly reduced OCRs in MCF7 cells (Figure 3C). OCR reductions followed the same trend in MCF7-HMGR cells treated with $1 \mathrm{mM} B M F$ (Figure 3E and 3F). To establish if BMF functions as a specific mitochondrial inhibitor only in cancer cells, we also performed a mitochondrial stress test on hTERT-BJ1 fibroblasts (Figure 3D), treated with BMF, Pravastatin or Simvastatin. Significant reductions in mitochondrial respiration were observed in hTERT-BJ1 fibroblasts treated with Pravastatin or Simvastatin (at $100 \mu \mathrm{M}$ and $1 \mathrm{mM}$ ), suggesting a toxic effect. However, no effects on mitochondrial respiration were observed in hTERT-BJ1 cells treated with BMF after 72 hours, indicating that the effect of BMF on mitochondrial respiration reduction is cell-type specific. Moreover, fatty acid oxidation (FAO) was also evaluated in MCF7 cells, under the same treatment conditions. This analysis revealed significant reductions in basal respiration, maximal respiration, and ATP levels, after palmitate addition, as compared to control cells (Figure $\mathbf{3} \mathbf{G}$ and $\mathbf{3 H}$ ), indicative of a decrease in exogenous FAO.

\subsection{BMF inhibits key signalling pathways involved in inflammation,} proliferation and "stemness"

502 To better understand its mechanism of action, we next examined the effects of BMF on several well-established signalling pathways, which have been shown to promote proliferation, inflammation and "stemness". For this purpose, we employed a panel of 
eight MCF7 reporter cell lines, engineered to carry validated luciferase constructs for monitoring the activation state of several distinct signalling networks, including: Sonic hedgehog, TGF $\beta$-SMAD, STAT3, Wnt, Interferon (IFN)- $\alpha / \beta-S T A T 1 / 2$, NRF2dependent antioxidant responses, IFN-Y-STAT1 and Notch pathways. Briefly, the MCF7 reporter cells were treated for 72 hours with $100 \mu \mathrm{M}$ and $1 \mathrm{mM}$ BMF. Note that BMF inhibited multiple CSC signaling pathways, including Wnt, IFN- $\alpha / \beta-S T A T 1 / 2$, STAT3, and Notch (Figure 4A, lower panel) and it activated IFN-Y-STAT1 and NRF2dependent antioxidant responses (Figure 4A, upper panel). However, no effects were observed for the Sonic hedgehog and TGF $\beta$-SMAD signaling pathways, after treatment with BMF (Fig 4A, upper panel).

\subsection{BMF reduces the secretion of inflammatory cytokines (IL-8 and GM-CSF)} It is well-established that Interleukin 8 (IL-8) [47] and Granulocyte-macrophage colony-stimulating factor (GM-CSF) [48] both stimulate malignant tumor cell growth and migration in vitro, as well as promote cancer progression in vivo [49, 50]. Thus, we next asked if BMF affects the release of these key inflammatory factors from MCF7 cancer cells. We detected GM-CSF (Figure 4B upper panel) and IL-8 levels (Figure 4B lower panel) in the cell culture media after $72 \mathrm{~h}$ of BMF and Pravastatin treatment, using GM-CSF and IL-8 ELISA kits. However, the levels of both secreted factors were significantly reduced in BMF-treated MCF7 cells, as compared to vehicle-alone control cells.

\subsection{BMF targets breast CSCs, by inhibiting HMGR and blocking mevalonate metabolism}

We provide several independent lines of evidence to directly support the idea that HMGR facilitates CSC propagation and mammosphere formation, via mevalonate metabolism. Firstly, MCF7 over-expressing HMGR show a greater efficiency towards mammosphere formation, as compared to vector-alone control cells generated in parallel (Figure 4C). Secondly, treatment with HMGR inhibitors (BMF or Pravastatin; at concentrations of $100 \mu \mathrm{M}$ and $1 \mathrm{mM}$ ) efficiently suppresses mammosphere formation, in both parental MCF7 cells, as well as in MCF7 cells over-expressing HMGR (Figure 4D and 4E). Thirdly, treatment with HMGR inhibitors (BMF or Pravastatin) was also sufficient to significantly decrease the ALDH-positive cell 
population by 2.5 -fold (Figure 4F). Importantly, ALDH-activity is an independent marker for "stemness" in cancer cells.

540 Finally, the addition of mevalonate to the tissue culture media was indeed sufficient to overcome the inhibitory effects of BMF and Pravastatin on i) mammosphere formation (Figure 4G) and ii) ALDH-activity (Figure 4H). However, the addition of exogenous cholesterol did not have the same rescue effect as mevalonate, indicating that mevalonate metabolism itself is critical for driving mammosphere formation and for maintaining CSC-activity, not the cholesterol end-product itself (Figure 4G and 4H).

\subsection{Rho-GDI-signalling is up-regulated in mammospheres treated with BMF,} driving a reduction in CSC propagation

To further mechanistically elucidate the down-stream effects of BMF on "stemness", we next used a "chemical" proteomics approach. Briefly, MCF7 cell mammospheres, formed after 72 hours pre-treatment with $1 \mathrm{mM} \mathrm{BMF}$, were harvested and subjected to proteomics analysis. These BMF-mammospheres were then directly compared with control monolayers, processed in parallel. Finally, all these proteomics data sets were used to generate a list of differentially expressed proteins, which was subjected to Ingenuity Pathway Analysis (IPA), to determine possible alterations in canonical pathways (Figure 5A) and toxicity functions (Figure 5B). Most notably, the Heat-Map shows that BMF-mammospheres behave in an opposite fashion, as compared with control MCF7 and T47D mammospheres, highlighting a complete change in terms of the regulation of numerous cancer-related, cell signaling pathways (Figure 5C). Importantly, canonical pathway analysis and the Heat-Map data clearly show that the Rho-GDI-signalling pathway is the only pathway activated in BMF-mammospheres, as compared with MCF7 and T47D mammosphere controls (Figure 5C and 5D). These results support and confirm the hypothesis that up-regulation of Rho-GDIsignalling clearly inhibits CSC propagation and mammosphere formation.

3.10. BMF reduces mevalonate formation, targeting breast CSCs through RhoGDI and RHOA/p27kip1 signalling

A defect in geranyl-geranylated proteins (GG) impairs small GTP-binding proteins, especially the RHO family of proteins. Geranyl-geranylated-pyrophosphate (GGPP) synthesis is necessary as an intermediate for the proper localization of RHO proteins 
572 to the cytoplasmic face of the cell membrane and their subsequent function. RHOA 573 regulates p27kip1 by mediating its phosphorylation on Thr-187 via CDK2 [51], resulting in the subsequent translocation of p27 from the nucleus to the cytosol, and thereby enhancing its degradation in the cytoplasm. In the absence of GGPP, through mevalonate inhibition by BMF, RHOA should be unable to carry out these functions and p27kip1 would therefore accumulate in the nucleus. Because p27kip1 is known to regulate stem cell self-renewal [52], we explored the role of RHOA/p27kip1 signalling in mediating the effects of BMF treatment on the CSC population. We assessed the impact of BMF treatment on RHOA inactivation by proteomics analysis. As expected, we observed that BMF treatment decreased the amount of RHOA and increased the amount of p27kip1 (Figure 6A). One mechanism by which BMF could suppress CSC self-renewal is through inhibition of RHOA and increased p27kip1 accumulation, which in turn would result in inhibition of CDK phosphorylation of RB, reducing both Cyclin $\mathrm{D}$ and Cyclin $\mathrm{E}$ expression. Therefore, we assessed the impact of BMF treatment on RHOA inactivation by measuring the levels of Cyclin $D$ and Cyclin E, as well as RB protein phosphorylation by Western blotting (Figure 6B). Cellular lysates from both BMF-treated MCF7 cells and vehicle-alone control MCF7 cells were partitioned into cytosolic fractions and immuno-blotted with antibodies involved in RHOA pathway regulation. As predicted, we observed that BMF treatment increased the amount of cytosolic p27kip1, and decreased cytosolic Cyclin-D and Cyclin-E, consistent with RHOA inhibition. These results are consistent with our observation that BMF results in arrest in the $\mathrm{G}_{0} / \mathrm{G}_{1}$ phase of the cell cycle. Moreover, these results confirm that BMF treatment perturbs cell cycle progression, through its ability to dys-regulate CCND1/p27/RB1/CCNE signalling. This pathway is highlighted schematically in Figure 6C.

\subsection{BMF down-regulates STAT1/3, as well as $\beta$-catenin protein expression} By proteomic analysis, we also validated that BMF regulates gene expression by reducing STAT1/3 and $\beta$-catenin protein levels. Our proteomic data shows a decreased amount of E-cadherin and CTK-receptors, as well as clear reductions in STAT1/3 and $\beta$-catenin protein expression (Figure 6A). The inhibition of these two pathways by BMF could suppress CSC self-renewal (Figure 6C). These findings are also consistent with our earlier results, using luciferase reporter constructs (Figure 
3.12. BMF down-regulates mitochondrial protein expression, mechanistically explaining the observed reductions in mitochondrial respiratory function To better understand the BMF-induced reductions in mitochondrial oxygen consumption, we further analysed our proteomic data sets. Our proteomic results suggest that BMF treatment may negatively impact mitochondrial respiration, by decreasing the amount of fatty-acyl-CoA and pyruvate inside the mitochondria, via reductions in CPT1 and the MPC transporter, consequently reducing acetyl-CoA formation. Moreover, the observed reduction of SLC25A1 transporter and the ACAT1 enzyme, are symptomatic of the inhibition of acetyl-CoA formation (Figure 6E). We also determined the impact of BMF on OXPHOS by measuring the protein levels of complexes I-V of the respiratory chain, by Western blotting (Figure 6D). As predicted, we observed that BMF treatment decreased the levels of complex I, II, IV and V, further validating the observed reductions in OCR and ATP production, as seen by Seahorse XFe96 analysis. These results are summarized schematically in Figure 6F.

\subsection{Prognostic value of HMGR in human breast cancer subtypes: Recurrence,} metastasis and overall survival

To assess the clinical relevance of HMGR, we also determined if HMGR mRNA transcript levels show any prognostic value, in human breast cancer patient cohorts, with long-term follow-up data (nearly 20 years). We analyzed both $E R(+)$ and ER(-) patient populations. Corresponding Kaplan-Meier (K-M) analysis curves are included in Figure 7 (See also Table S1 and Table S2). Note that high mRNA levels of HMGR show an association with reduced relapse-free survival (RFS), i.e., higher tumor recurrence. More specifically, HMGR had prognostic value in both: i) $E R(+)$ patients, normally treated with endocrine therapy and ii) ER(-) patients, consistently treated with chemotherapy. Interestingly, HMGR was especially predictive in the following more aggressive breast cancer groups: i) $E R(+) / L u m i n a l ~ B$ and ii) $E R(-) / B a s a l$ subtypes. High mRNA levels of HMGR were also associated with increased distant metastasis (DMFS) and poor overall survival (OS).

\section{Discussion}

638 Targeting CSCs is a new promising field for anti-cancer therapy [53]. Several studies 
have recently highlighted a strong association between i) metabolism and ii) CSCs biology [15]. In order to target CSCs, it will be necessary to take into account several additional parameters, including tumor heterogeneity. For example, it is now wellaccepted that CSCs are somehow dependent on cancer-promoting mutations and this ultimately produces several different sub-populations of 'progenitor' cells, as well as 'mature' or 'differentiated' cancer cells [10, 13].

One new promising class of anti-CSC drugs are the statins. They are competitive inhibitors of HMGR, a key enzyme required for cholesterol biosynthesis. Statins can inhibit human tumor growth, by decreasing the local synthesis of cholesterol. Indeed, rapidly growing tumor cells require high levels of cholesterol content, as an essential component of their cellular membranes. As a consequence, many cancer patients actually have reduced plasma levels of cholesterol. Interestingly, HMGR inhibition, by the statins, also depletes several other metabolic intermediates that may be involved in CSC propagation, such as mevalonate $[54,55]$.

Although statins are very effective as anti-cholesterolemic drugs, they suffer from a number of common side-effects, including muscle wasting and damage (both skeletal and cardiac). As a consequence, many scientists are currently searching for new statin-like molecules, that show anti-cancer properties, but lack the side-effects of commercial statins [56]. Here, we evaluated the possibility that Brutieridin and Melitidin, two statin-like flavanone inhibitors of HMGR, extracted from Bergamot fruit, exert a similar behavior with respect to the commercial statins (Simvastatin and Pravastatin), to prevent cancer progression and CSC propagation.

Using unbiased label-free proteomics analysis, we identified specific protein data sets related to CSC propagation. More specifically, we identified proteins that were specifically up-regulated in human breast cancer cells, when cultured under anchorage-independent growth conditions. These conditions greatly facilitate the formation of mammospheres or 3D-tumor-spheres, thereby substantially enriching the CSC population. Bio-informatic analysis of these MCF7-mammosphere protein data sets revealed the up-regulation of enzymes that are characteristic of cholesterol biosynthesis and mevalonate metabolism, including HMGR itself. Virtually identical results were also obtained with T47D-mammospheres, highlighting the conserved role of mevalonate metabolism in CSC propagation. In accordance with these findings, we showed that BMF effectively reduces HMGR activity and blocks mammosphere formation. Treatment with BMF also reduced the growth of MCF7 
673 cells, leading to arrest in the $\mathrm{G}_{0} / \mathrm{G}_{1}$ phase of the cell cycle. In this context, BMF 674 behaved similarly to the commercial statins; however, BMF did not show the same side-effect profile. Remarkably, while the commercial statins showed substantial toxicity, BMF was non-toxic when applied to normal human fibroblasts (hTERT-BJ1 cells). Therefore, BMF may represent a non-toxic alternative to the commercial statins.

679 To pinpoint which CSC pathways were targeted by BMF, we used a panel of isogenic MCF7 cell lines, harboring a series of luciferase reporter constructs; this panel of MCF7 cell lines was generated to quantitatively measure the activation state of 8 different signalling cascades or networks. Interestingly, BMF treatment inhibited several distinct CSC signaling pathways, including: STAT1/3, Notch and Wnt/Betacatenin. In addition, BMF also stimulated the anti-oxidant response, triggering the activation of both NRF2- and IFN- $\alpha / \beta-S T A T 1 / 2$ signalling.

We also measured the metabolic effects of BMF on cancer cells (MCF7) and normal fibroblasts (hTERT-BJ1), using the Seahorse XFe96 metabolic flux analyzer. Importantly, BMF significantly inhibited the oxygen consumption rate (OCR) and ATP production in MCF7 cells; virtually identical results were obtained with commercial statins. However, BMF did not show any effects on mitochondrial respiration in normal human fibroblasts, while commercial statins still showed strong inhibition of mitochondrial function. Thus, the mitochondrial effects of BMF appear to be specific to cancer cells. Inflammatory cytokines play a major role in tumor progression and metastasis. For example, these inflammatory cytokines (i.e., IL-8 and GM-CSF) promote tumor invasive properties [57] and activate CSC signalling pathways, including those regulated by Wnt, Notch and STAT1/3 $[58,59]$. As a consequence we evaluated the effects of BMF on cytokine release from MCF7 cells into the culture media. Interestingly, our results directly show that BMF significantly inhibited the release of both GM-CSF and IL-8, in a dose-dependent manner.

Using a specific CSC marker (ALDH-activity), we also showed that BMF treatment significantly decreased the ALDH-positive cell population in MCF7 cells. Moreover, the addition of mevalonate, the product of the HMGR enzyme, to the culture medium rescued the CSC population, with a complete restoration of the ALDEFLUOR-positive population. However, cholesterol did not have the same rescue effect. Therefore, 
these results directly validate the idea that mevalonate is an essential metabolite for driving CSC propagation, but that this is unrelated to cholesterol biosynthesis itself. Further proteomics analysis also allowed us to dissect the mechanism by which BMF inhibits cell proliferation and induces arrest in the $\mathrm{G}_{0} / \mathrm{G}_{1}$ phase of the cell cycle. In particular, BMF up-regulated Rho-GDI-signalling, leading to dys-regulation along the CCND1/p27/ RB1/CCNE pathway.

\section{Conclusion}

In summary, our current results directly show that BMF is a natural, non-toxic, inhibitor of HMGR, that can be effectively used to target mitochondrial metabolism (OXPHOS) and fatty acid oxidation (FAO) in breast cancer cells, preventing the CSCs formation and their propagation via Rho-GDI-signalling.

\section{Author contributions}

MF, AC, FS and MPL conceived and initiated this project. MF performed most experiments, analyze the data and generated the final figures. MPP and RSA performed some experiments. LB, LDD, VD, GS and AC purified and characterized the BMF compounds. MF wrote the first draft of the manuscript, which was then further edited by all the co-authors, especially by FS and MPL.

\section{Acknowledgments}

No conflicts of interest to declare

\section{References}

[1] D.A. Berry, K.A. Cronin, S.K. Plevritis, D.G. Fryback, L. Clarke, M. Zelen, J.S. Mandelblatt, A.Y. Yakovlev, J.D. Habbema, E.J. Feuer, I. Cancer, C. Surveillance Modeling Network, Effect of screening and adjuvant therapy on mortality from breast cancer, The New England journal of medicine, 353 (2005) 1784-1792.

[2] Z. Mei, M. Liang, L. Li, Y. Zhang, Q. Wang, W. Yang, Effects of statins on cancer mortality and progression: A systematic review and meta-analysis of 95 cohorts including 1,111,407 individuals, Int J Cancer, 140 (2017) 1068-1081. 
737

738

739

740

741

742

743

744

745

746

747

748

749

750

751

752

753

754

755

756

757

758

759

760

761

762

763

764

765

766

767

768

769

[3] T.P. Ahern, T.L. Lash, P. Damkier, P.M. Christiansen, D.P. Cronin-Fenton, Statins and breast cancer prognosis: evidence and opportunities, Lancet Oncol, 15 (2014) e461-468.

[4] S. Pisanti, P. Picardi, E. Ciaglia, A. D'Alessandro, M. Bifulco, Novel prospects of statins as therapeutic agents in cancer, Pharmacological research, 88 (2014) 84-98. [5] B. Yeganeh, E. Wiechec, S.R. Ande, P. Sharma, A.R. Moghadam, M. Post, D.H. Freed, M. Hashemi, S. Shojaei, A.A. Zeki, S. Ghavami, Targeting the mevalonate cascade as a new therapeutic approach in heart disease, cancer and pulmonary disease, Pharmacology \& therapeutics, 143 (2014) 87-110.

[6] K. Tanaka, H. Osada, Y. Murakami-Tonami, Y. Horio, T. Hida, Y. Sekido, Statin suppresses Hippo pathway-inactivated malignant mesothelioma cells and blocks the YAP/CD44 growth stimulatory axis, Cancer Lett, 385 (2017) 215-224.

[7] C. Ginestier, F. Monville, J. Wicinski, O. Cabaud, N. Cervera, E. Josselin, P. Finetti, A. Guille, G. Larderet, P. Viens, S. Sebti, F. Bertucci, D. Birnbaum, E. CharafeJauffret, Mevalonate metabolism regulates Basal breast cancer stem cells and is a potential therapeutic target, Stem cells, 30 (2012) 1327-1337.

[8] C.T. Jordan, M.L. Guzman, M. Noble, Cancer stem cells - Reply, New Engl J Med, 355 (2006) 2703-2703.

[9] T. Reya, S.J. Morrison, M.F. Clarke, I.L. Weissman, Stem cells, cancer, and cancer stem cells, Nature, 414 (2001) 105-111.

[10] J.A. Magee, E. Piskounova, S.J. Morrison, Cancer stem cells: impact, heterogeneity, and uncertainty, Cancer cell, 21 (2012) 283-296.

[11] C.T. Jordan, M.L. Guzman, M. Noble, Mechanisms of disease - Cancer stem cells, New Engl J Med, 355 (2006) 1253-1261.

[12] G. Dontu, M. Al-Hajj, W.M. Abdallah, M.F. Clarke, M.S. Wicha, Stem cells in normal breast development and breast cancer, Cell proliferation, 36 Suppl 1 (2003) 59-72.

[13] M.D. Brooks, M.L. Burness, M.S. Wicha, Therapeutic Implications of Cellular Heterogeneity and Plasticity in Breast Cancer, Cell Stem Cell, 17 (2015) 260-271.

[14] C. Ginestier, E. Charafe-Jauffret, D. Birnbaum, Targeting breast cancer stem cells: fishing season open!, Breast cancer research : BCR, 12 (2010) 312.

[15] M. Peiris-Pages, U.E. Martinez-Outschoorn, R.G. Pestell, F. Sotgia, M.P. Lisanti, Cancer stem cell metabolism, Breast cancer research : BCR, 18 (2016) 55. 
770

771

772

773

774

775

776

777

778

779

780

781

782

783

784

785

786

787

788

789

790

791

792

793

794

795

796

797

798

799

800

801

802

[16] K. Gauthaman, C.Y. Fong, A. Bongso, Statins, stem cells, and cancer, J Cell Biochem, 106 (2009) 975-983.

[17] P. Jiang, R. Mukthavaram, Y. Chao, N. Nomura, I.S. Bharati, V. Fogal, S. Pastorino, D. Teng, X. Cong, S.C. Pingle, S. Kapoor, K. Shetty, A. Aggrawal, S. Vali, T. Abbasi, S. Chien, S. Kesari, In vitro and in vivo anticancer effects of mevalonate pathway modulation on human cancer cells, Br J Cancer, 111 (2014) 1562-1571.

[18] M. Thurnher, G. Gruenbacher, O. Nussbaumer, Regulation of mevalonate metabolism in cancer and immune cells, Biochimica et biophysica acta, 1831 (2013) 1009-1015.

[19] J. Greenwood, L. Steinman, S.S. Zamvil, Statin therapy and autoimmune disease: from protein prenylation to immunomodulation, Nat Rev Immunol, 6 (2006) 358-370.

[20] S.J. Malachowski, A.M. Quattlebaum, B. Miladinovic, Adverse Effects of Statins, JAMA, 317 (2017) 1079-1080.

[21] P. du Souich, G. Roederer, R. Dufour, Myotoxicity of statins: Mechanism of action, Pharmacology \& therapeutics, (2017).

[22] S.H. Lin, K.J. Huang, C.F. Weng, D. Shiuan, Exploration of natural product ingredients as inhibitors of human HMG-CoA reductase through structure-based virtual screening, Drug Des Devel Ther, 9 (2015) 3313-3324.

[23] J.B. Harborne, C.A. Williams, Advances in flavonoid research since 1992, Phytochemistry, 55 (2000) 481-504.

[24] A.M. Patti, P.P. Toth, R.V. Giglio, M. Banach, M. Noto, D. Nikolic, G. Montalto, M. Rizzo, Nutraceuticals as an Important Part of Combination Therapy in Dyslipidaemia, Curr Pharm Des, (2017).

[25] O. Benavente-Garcia, J. Castillo, M. Alcaraz, V. Vicente, J.A. Del Rio, A. Ortuno, Beneficial action of Citrus flavonoids on multiple cancer-related biological pathways, Curr Cancer Drug Targets, 7 (2007) 795-809.

[26] D. Yin, J. Li, X. Lei, Y. Liu, Z. Yang, K. Chen, Antiviral Activity of Total Flavonoid Extracts from Selaginella moellendorffii Hieron against Coxsackie Virus B3 In Vitro and In Vivo, Evidence-based complementary and alternative medicine : eCAM, 2014 (2014) 950817.

[27] G. Mandalari, R.N. Bennett, G. Bisignano, D. Trombetta, A. Saija, C.B. Faulds, M.J. Gasson, A. Narbad, Antimicrobial activity of flavonoids extracted from bergamot 
803 (Citrus bergamia Risso) peel, a byproduct of the essential oil industry, Journal of

804

805

806

807

808

809

810

811

812

813

814

815

816

817

818

819

820

821

822

823

824

825

826

827

828

829

830

831

832

833

834

835 applied microbiology, 103 (2007) 2056-2064.

[28] H.P. Kim, K.H. Son, H.W. Chang, S.S. Kang, Anti-inflammatory plant flavonoids and cellular action mechanisms, Journal of pharmacological sciences, 96 (2004) 229245.

[29] I. Peluso, C. Miglio, G. Morabito, F. loannone, M. Serafini, Flavonoids and immune function in human: a systematic review, Critical reviews in food science and nutrition, 55 (2015) 383-395.

[30] G. Gattuso, D. Barreca, C. Gargiulli, U. Leuzzi, C. Caristi, Flavonoid composition of Citrus juices, Molecules, 12 (2007) 1641-1673.

[31] Y. Nogata, K. Sakamoto, H. Shiratsuchi, T. Ishii, M. Yano, H. Ohta, Flavonoid composition of fruit tissues of citrus species, Biosci Biotechnol Biochem, 70 (2006) 178-192.

[32] A.R. Cappello, V. Dolce, D. lacopetta, M. Martello, M. Fiorillo, R. Curcio, L. Muto, D. Dhanyalayam, Bergamot (Citrus bergamia Risso) Flavonoids and Their Potential Benefits in Human Hyperlipidemia and Atherosclerosis: an Overview, Mini Rev Med Chem, 16 (2016) 619-629.

[33] L. Di Donna, G. De Luca, F. Mazzotti, A. Napoli, R. Salerno, D. Taverna, G. Sindona, Statin-like principles of bergamot fruit (Citrus bergamia): isolation of 3hydroxymethylglutaryl flavonoid glycosides, J Nat Prod, 72 (2009) 1352-1354.

[34] L. Di Donna, D. lacopetta, A.R. Cappello, G. Gallucci, E. Martello, M. Fiorillo, V. Dolce, G. Sindona, Hypocholesterolaemic activity of 3-hydroxy-3-methyl-glutaryl flavanones enriched fraction from bergamot fruit (Citrus bergamia): "In vivo" studies, J Funct Foods, 7 (2014) 558-568.

[35] C. Ginestier, M.S. Wicha, Mammary stem cell number as a determinate of breast cancer risk, Breast cancer research : BCR, 9 (2007) 109.

[36] C. Ginestier, M.H. Hur, E. Charafe-Jauffret, F. Monville, J. Dutcher, M. Brown, J. Jacquemier, P. Viens, C.G. Kleer, S. Liu, A. Schott, D. Hayes, D. Birnbaum, M.S. Wicha, G. Dontu, ALDH1 is a marker of normal and malignant human mammary stem cells and a predictor of poor clinical outcome, Cell Stem Cell, 1 (2007) 555-567.

[37] A. Carnero, Y. Garcia-Mayea, C. Mir, J. Lorente, I.T. Rubio, L.L. ME, The cancer stem-cell signaling network and resistance to therapy, Cancer Treat Rev, 49 (2016) 25-36. 
[38] S. Liu, G. Dontu, M.S. Wicha, Mammary stem cells, self-renewal pathways, and carcinogenesis, Breast cancer research : BCR, 7 (2005) 86-95.

[39] P. Skehan, R. Storeng, D. Scudiero, A. Monks, J. McMahon, D. Vistica, J.T. Warren, H. Bokesch, S. Kenney, M.R. Boyd, New colorimetric cytotoxicity assay for anticancer-drug screening, J Natl Cancer Inst, 82 (1990) 1107-1112.

[40] R. Balachandran, E. ter Haar, J.C. Yalowich, M.J. Welsh, S.G. Grant, B.W. Day, Induction of human breast cancer cell apoptosis from G2/M preceded by stimulation into the cell cycle by Z-1,1-dichloro-2,3-diphenylcyclopropane, Biochem Pharmacol, 57 (1999) 97-110.

[41] M. Fiorillo, R. Lamb, H.B. Tanowitz, A.R. Cappello, U.E. Martinez-Outschoorn, F. Sotgia, M.P. Lisanti, Bedaquiline, an FDA-approved antibiotic, inhibits mitochondrial function and potently blocks the proliferative expansion of stem-like cancer cells (CSCs), Aging (Albany NY), 8 (2016) 1593-1607.

[42] M. Fiorillo, R. Lamb, H.B. Tanowitz, L. Mutti, M. Krstic-Demonacos, A.R. Cappello, U.E. Martinez-Outschoorn, F. Sotgia, M.P. Lisanti, Repurposing atovaquone: targeting mitochondrial complex III and OXPHOS to eradicate cancer stem cells, Oncotarget, 7 (2016) 34084-34099.

[43] S.M. Johnson, C. Dempsey, A. Chadwick, S. Harrison, J. Liu, Y. Di, O.J. McGinn, M. Fiorillo, F. Sotgia, M.P. Lisanti, M. Parihar, S. Krishnan, V. Saha, Metabolic reprogramming of bone marrow stromal cells by leukemic extracellular vesicles in acute lymphoblastic leukemia, Blood, 128 (2016) 453-456.

[44] M. Peiris-Pages, F. Sotgia, M.P. Lisanti, Chemotherapy induces the cancerassociated fibroblast phenotype, activating paracrine Hedgehog-GLI signalling in breast cancer cells, Oncotarget, 6 (2015) 10728-10745.

[45] F.L. Shaw, H. Harrison, K. Spence, M.P. Ablett, B.M. Simoes, G. Farnie, R.B. Clarke, A detailed mammosphere assay protocol for the quantification of breast stem cell activity, J Mammary Gland Biol Neoplasia, 17 (2012) 111-117.

[46] M. Peiris-Pages, D.L. Smith, B. Gyorffy, F. Sotgia, M.P. Lisanti, Proteomic identification of prognostic tumour biomarkers, using chemotherapy-induced cancerassociated fibroblasts, Aging (Albany NY), 7 (2015) 816-838.

[47] D.J. Waugh, C. Wilson, The interleukin-8 pathway in cancer, Clinical cancer research : an official journal of the American Association for Cancer Research, 14 (2008) 6735-6741. 
[48] E. Van Overmeire, B. Stijlemans, F. Heymann, J. Keirsse, Y. Morias, Y. Elkrim, L. Brys, C. Abels, Q. Lahmar, C. Ergen, L. Vereecke, F. Tacke, P. De Baetselier, J.A. Van Ginderachter, D. Laoui, M-CSF and GM-CSF Receptor Signaling Differentially Regulate Monocyte Maturation and Macrophage Polarization in the Tumor Microenvironment, Cancer Res, 76 (2016) 35-42. [49] N. Shao, Z. Lu, Y. Zhang, M. Wang, W. Li, Z. Hu, S. Wang, Y. Lin, Interleukin-8 upregulates integrin beta3 expression and promotes estrogen receptor-negative breast cancer cell invasion by activating the PI3K/Akt/NF-kappaB pathway, Cancer Lett, 364 (2015) 165-172.

[50] M. Waghray, M. Yalamanchili, M. Dziubinski, M. Zeinali, M. Erkkinen, H. Yang, K.A. Schradle, S. Urs, M. Pasca Di Magliano, T.H. Welling, P.L. Palmbos, E.V. Abel, V. Sahai, S. Nagrath, L. Wang, D.M. Simeone, GM-CSF Mediates MesenchymalEpithelial Cross-talk in Pancreatic Cancer, Cancer Discov, 6 (2016) 886-899. [51] A. Kazi, A. Carie, M.A. Blaskovich, C. Bucher, V. Thai, S. Moulder, H. Peng, D. Carrico, E. Pusateri, W.J. Pledger, N. Berndt, A. Hamilton, S.M. Sebti, Blockade of protein geranylgeranylation inhibits Cdk2-dependent p27Kip1 phosphorylation on Thr187 and accumulates p27Kip1 in the nucleus: implications for breast cancer therapy, Mol Cell Biol, 29 (2009) 2254-2263.

[52] A. Besson, H.C. Hwang, S. Cicero, S.L. Donovan, M. Gurian-West, D. Johnson, B.E. Clurman, M.A. Dyer, J.M. Roberts, Discovery of an oncogenic activity in p27Kip1 that causes stem cell expansion and a multiple tumor phenotype, Genes Dev, 21 (2007) 1731-1746.

[53] S. Liu, M.S. Wicha, Targeting breast cancer stem cells, Journal of clinical oncology : official journal of the American Society of Clinical Oncology, 28 (2010) 4006-4012.

[54] K. Gauthaman, N. Manasi, A. Bongso, Statins inhibit the growth of variant human embryonic stem cells and cancer cells in vitro but not normal human embryonic stem cells, Br J Pharmacol, 157 (2009) 962-973.

[55] S. Sorscher, Clinical confirmation of an anti-metastasis effect from statins, Int J Biochem Cell Biol, 57 (2014) 207. anti-metastatic and anti-tumorigenic effects in ovarian cancer, Oncotarget, (2015). 
[57] C. Ginestier, S. Liu, M.E. Diebel, H. Korkaya, M. Luo, M. Brown, J. Wicinski, O. Cabaud, E. Charafe-Jauffret, D. Birnbaum, J.L. Guan, G. Dontu, M.S. Wicha, CXCR1 blockade selectively targets human breast cancer stem cells in vitro and in xenografts, The Journal of clinical investigation, 120 (2010) 485-497.

[58] M. Kakarala, D.E. Brenner, H. Korkaya, C. Cheng, K. Tazi, C. Ginestier, S. Liu, G. Dontu, M.S. Wicha, Targeting breast stem cells with the cancer preventive compounds curcumin and piperine, Breast cancer research and treatment, 122 (2010) 777-785.

[59] S.S. Chung, J.V. Vadgama, Curcumin and epigallocatechin gallate inhibit the cancer stem cell phenotype via down-regulation of STAT3-NFkappaB signaling, Anticancer research, 35 (2015) 39-46.

\section{FIGURE LEGENDS}

Figure 1. Brutieridin (B) and Melitidin (M) are natural flavonoids (F): Extraction, purification and inhibition of HMGR activity.

(A) Schematic work-flow diagram illustrating the extraction and purification of Brutieridin and Melitidin flavonoids (BMF). SPE $=$ solid-phase extraction, MPLC = medium-pressure liquid-chromatography, $\mathrm{HPLC}=$ high-pressure liquidchromatography.

(B) The detailed chemical structures of Brutieridin and Melitidin are shown.

(C) HPLC/UV Chromatogram of BMF. The molecules shown are Brutieridin (1, r.t. 41.64) and Melitidin (2, r.t. 40.06).

(D) BMF inhibits HMGR activity. The effects of BMF on HMGR activity was tested using a well-established assay kit (Sigma-Aldrich). Simvastatin and Pravastatin were used as positive controls (not shown).

Figure 2. BMF differentially affects the viability of human breast cancer cells (MCF7) and normal fibroblasts (hTERT-BJ1).

(A) Generating MCF7-HMGR cells. Parental MCF7 cells were stably-transduced with a lentiviral vector encoding HMGR or an empty vector control (EV). Recombinant over-expression of HMGR in MCF7 cells was confirmed by immune-blot analysis, with specific antibody probes. The expression of $\beta$-actin was assessed to ensure equal protein loading. 
(B) Effects of statin-like molecules on cell viability. The effects of BMF, Pravastatin and Simvastatin were evaluated using cultures of human breast cancer cells (MCF7 or MCF7-HMGR) or normal human fibroblasts (hTERT-BJ1). Cells were treated for 72 or 120 hours with $100 \mu \mathrm{M}$ and $1 \mathrm{mM}$ BMF (upper panel), $100 \mu \mathrm{M}$ and $1 \mathrm{mM}$ Pravastatin (middle panel), 10, 50, $100 \mu \mathrm{M}$ and $1 \mathrm{mM}$ Simvastatin (lower panel). Cell viability was assessed using the SRB assay to measure protein content and was expressed as a percentage of control cells (vehicle-alone treated cells).

(C) BMF induces cell cycle arrest in MCF7 cells. Results are expressed as the percentage of cells found in different phases of the cell cycle. Note that BMF and Pravastatin both impair the G1/S transition of MCF7 breast cancer cells.

The values shown represent the means \pm S.E.M. of three biological replicates from three independent experiments. Statistical differences compared to control are given as: ${ }^{* *} p \leq 0.001$ or ${ }^{* *} p \leq 0.0001$, using the one-way ANOVA t-test.

Figure 3. BMF differentially effects mitochondrial respiration in human breast cancer cells (MCF7 and MCF7-HMGR) and normal human fibroblasts (hTERTBJ1).

(A and B) Analyzing the metabolic phenotype of MCF7-HMGR cells. MCF7HMGR cells and corresponding vector alone control cells (MCF7-EV), were subjected to metabolic flux analysis, with the Seahorse XFe96. The extracellular acidification rate (A) (ECAR; a measure of glycolytic flux) and (B) oxygen consumption rate (OCR; a measure of mitochondrial respiration) were assessed. Note that recombinant overexpression of HMGR in MCF7 cells had no effect on ECAR, but significantly increased OCR, which is indicative of increased mitochondrial respiration.

(C) Note that BMF treatment decreases mitochondrial respiration in MCF7 breast cancer cells. Significant reductions in mitochondrial respiration were observed, experimentally, in MCF7 cell monolayers treated for 72 hours with BMF or Pravastatin/Simvastatin (100 $\mu \mathrm{M}$ and $1 \mathrm{mM}$ each).

(D) Note that BMF treatment does not effect mitochondrial respiration in normal human fibroblasts (hTERT-BJ1 cells). However, Pravastatin and Simvastatin (100 $\mu \mathrm{M}$ and $1 \mathrm{mM}$ each) both significantly inhibited mitochondrial respiration in hTERT-BJ1 cells. Tracings from 3 independent experiments are shown for each experimental 967 condition. 
(E) Significant reductions in maximal respiration, ATP production, and spare respiratory capacity were observed experimentally in MCF7 cells treated with $1 \mathrm{mM}$ BMF, compared to the vehicle-alone treated control.

(F) BMF treatment also significantly reduces ATP production, basal respiration, maximal respiration and spare respiratory capacity, in MCF7-HMGR cells.

(G) BMF treatment reduces the fatty acid oxidation (FAO) profile in MCF7 breast cancer cells. Significant reductions in basal respiration, maximal respiration, and ATP levels were observed experimentally, in treated MCF7 cells, after palmitate addition, compared to untreated cells.

In panels $A-G$, merged tracings of 3 independent experiments are shown for each experimental condition. ${ }^{*} p<0.01 ;{ }^{* *} p<0.001 ;{ }^{* \star} p<0.0001$, one-way ANOVA t-test.

\section{Figure 4: BMF treatment effectively inhibits CSC signalling and propagation:} Rescue with the simple metabolite Mevalonate.

(A) BMF treatment inhibits signalling pathways related to "stemness" and inflammation, while inducing the anti-oxidant response. Note that BMF treatment inhibits the following four pathways related to CSC signalling: Wnt/beta-catenin, IFN$\alpha / \beta-S T A T 1 / 2$, STAT3, as well as, Notch (lower panel). In contrast, BMF treatment activates IFN- $\gamma$-STAT1 signalling and the NRF2-dependent anti-oxidant response (upper panel). However, BMF had no effects on TGF $\beta$-SMAD and Sonic hedgehog signalling (upper panel). ${ }^{*} p<0.01 ;{ }^{* *} p<0.001 ;{ }^{* * *} p<0.0001$, using the Student's $\mathrm{t}$ test.

(B) BMF reduces the secretion of inflammatory cytokines. MCF7 cells were treated with $100 \mu \mathrm{M}$ and $1 \mathrm{mM}$ of BMF or Pravastatin after 72 hours. Afterwards, the cell culture media was collected and the levels of secreted GM-CSF and IL-8 were determined using an ELISA test. ${ }^{*} p<0.01$; ${ }^{* *} p<0.001$, evaluated with one-way ANOVA.

(C) HMGR over-expression elevates 3D-spheroid formation. Note that MCF7-HMGR cells showed the highest mammosphere formation efficiency (MFE). ${ }^{* *} p<0.001$, evaluated with the Student's t-test.

(D and E) Note that BMF-treatment dose-dependently inhibited 3D-mammosphere formation, in both MCF7-HMGR and MCF7-EV cells. Virtually identical results were also obtained when BMF or Pravastatin was added directly to the mammosphere culture media, without any monolayer pre-treatment. ${ }^{*} p<0.01 ;{ }^{* *} / \bullet p<0.001 ;{ }^{* * *} / \bullet \bullet$ 
$p<0.0001$, evaluated with one-way ANOVA. MFE (mammosphere formation 1003 efficiency) is shown and was normalized to $100 \%$.

1004 (F) BMF treatment inhibits ALDH-activity. MCF7 cells were pre-treated with BMF or 1005 Pravastatin (100 $\mathrm{\mu M}$ and $1 \mathrm{mM}$ each) as monolayers for 48 hours and then assessed 1006 for ALDEFLUOR-activity, as an independent marker of CSCs. Note that treatment 1007 with BMF or Pravastatin decreases the ALDH-positive cell population. ${ }^{*} p<0.01 ;{ }^{* *} p$ $1008<0.001 ;{ }^{* *} p<0.0001$, evaluated with one-way ANOVA.

1009 (G) Mevalonate restores 3D-spheroid formation, after inhibition with BMF or 1010 Pravastatin. First, MCF-7 cells were seeded into low-attachment plates for assessing $3 \mathrm{D}$-mammosphere formation, in the presence of BMF or pravastatin (100 $\mu \mathrm{M}$ and 1 $\mathrm{mM}$ each). Then, specific metabolites [either: i) Mevalonate (1 $\mathrm{mM}$ ) or ii) Cholesterol $(10 \mu \mathrm{M})]$ were added, to determine if they could reverse the inhibitory effects of BMF and Pravastatin. Note that Mevalonate treatment was indeed sufficient to revert the inhibitory effect and restore 3D-mammosphere formation, while cholesterol was ineffective. MFE (mammosphere formation efficiency) is shown and was normalized to $100 \%$. ${ }^{*} p<0.01,{ }^{* *} p<0.001^{* * *}, p<0.0001$, evaluated with one-way ANOVA.

(H) Mevalonate restores ALDH-activity, after inhibition with BMF or Pravastatin. Note that Mevalonate treatment was indeed sufficient to revert the inhibitory effect and restore ALDH-activity, while cholesterol was ineffective. ${ }^{* \star *} p<0.0001$, evaluated with one-way ANOVA.

(I) Schematic diagram illustrating the key steps involved in mevalonate metabolism and cholesterol biosynthesis, which appear to be involved in CSC propagation.

Figure 5: Ingenuity Pathway Analysis (IPA) of proteomics data sets obtained from human breast cancer cells, grown as 3D-spheroids, but pre-treated with BMF.

(A) Canonical pathways predicted to be altered by BMF in 3D-spheroids are shown. Briefly, MCF7 cells were pre-treated as a monolayer with BMF (1 mM) for $72 \mathrm{~h}$; then the cells were harvested and allowed to undergo 3D-mammosphere formation. In parallel, MCF7 cells were grown as a vehicle-alone treated monolayer. Then, comparative proteomics analysis was performed, essentially as outlined in Figure S1, where BMF-spheroids (S) were compared with control monolayer (M) cells. As expected, certain canonical pathways were significantly altered by the differential 
protein expression in MCF7 3D-spheres pre-treated with BMF $(p<0.05)$. The $p$-value for each pathway is indicated by the bar and is expressed as -1 times the log of the $p$ value. A positive z-score (Orange color; z-score > 1.9) represents the up-regulation of a specific pathway, while a negative z-score (Blue color; z-score $<-1.9$ ) indicates the down-regulation of a pathway.

(B) Toxicity effects of differentially expressed proteins in MCF7 3D-spheres (S) pretreated with BMF, relative to control monolayer cells $(\mathrm{M})$, are shown. Ingenuity Pathway Analysis showed that certain toxicity functions are significantly enriched by the proteins differentially expressed in this comparative analysis $(p<0.05)$. In the Bar chart, the $p$-value for each pathway is indicated by the bar and is expressed as -1 times the log of the $p$-value (cutoff $z$-score \pm 2 ).

\section{6}

(C) HeatMap of the canonical pathways predicted to be altered in 3D-spheres (S); T47D, MCF7 and BMF-treated MCF7), all relative to monolayer cells (M). A positive z-score (Orange) points towards the activation of a pathway, while a negative z-score (Blue) indicates the inhibition of a pathway. Therefore, note that Rho-GDI-signaling is normally "inhibited" in T47D and MCF7 3D-spheres, while the same pathway is "activated" by BMF-pretreatment in MCF7 cells (Boxed in Red).

(D) HeatMap of the key regulators identified by proteomics analysis that are either increased (Orange) or decreased (Blue), when 3D-spheres (S); from T47D, MCF7 and BMF-treated MCF7) are compared, all relative to monolayer cells (M).

Figure 6: Pleiotropic effects of BMF on signalling, cell cycle progression and mitochondrial protein expression: Proteomic evidence and validation. (A-C) Rho-GDI, RHOA/p27Kip1, STAT1/3 and $\beta$-catenin signalling. (A) Proteomics analysis: A selection of MCF7 proteins related to Rho-GDI, RHOA/p27Kip1, STAT1/3 and $\beta$-catenin signalling, that show altered expression in response to BMF pre-treatment, are shown. (B) Proteomics validation: Immunoblot analysis was used to validate and confirm our results from the proteomics analysis of MCF7 3D-spheroids, prepared from cells pre-treated with BMF. For example, note that BMF induces p27Kip1 and reduces Cyclin E expression. (C) Summary diagram: This illustration highlights the effects of BMF on mevalonate pathway synthesis, Rho-GDI signalling and a variety of nuclear events that control 1067 cell proliferation. 

validation: Immuno-blot analysis was used to validate and confirm our results from the proteomics analysis of MCF7 3D-spheroids, prepared from cells pre-treated with BMF. For example, note that various OXPHOS complex members were downregulated upon BMF-treatment. (E) Proteomics analysis: A selection of MCF7 proteins related to mitochondrial respiration (OXPHOS) and function, that show reduced expression in response to BMF pre-treatment, are shown. (F) Summary diagram: This illustration mechanistically highlights the effects of BMF on mitochondrial OXPHOS and fatty acid oxidation (FAO).

Figure 7: Prognostic value of HMGR in human breast cancer sub-types.

To assess the clinical relevance of HMGR, we also determined if HMGR mRNA transcript levels show any prognostic value, in human breast cancer patient cohorts, with long-term follow-up data (nearly 20 years). We analyzed both $\operatorname{ER}(+)$ and $E R(-)$ patient populations. Note that high mRNA levels of HMGR show an association with reduced relapse-free survival (RFS), i.e., higher tumor recurrence. (A) All breast cancers and $E R(+)$ sub-types are shown; (B) ER(-) breast cancer sub-types are shown. More specifically, HMGR had prognostic value in both: i) $E R(+)$ patients, normally treated with endocrine therapy and ii) ER(-) patients, consistently treated with chemotherapy. Interestingly, HMGR was especially predictive in the following more aggressive breast cancer groups: i) $E R(+) / L u m i n a l ~ B$ and ii) $E R(-) / B a s a l$ subtypes. High mRNA levels of HMGR were also associated with increased distant metastasis (DMFS) and poor overall survival (OS) (See also Tables S1 and S2). 


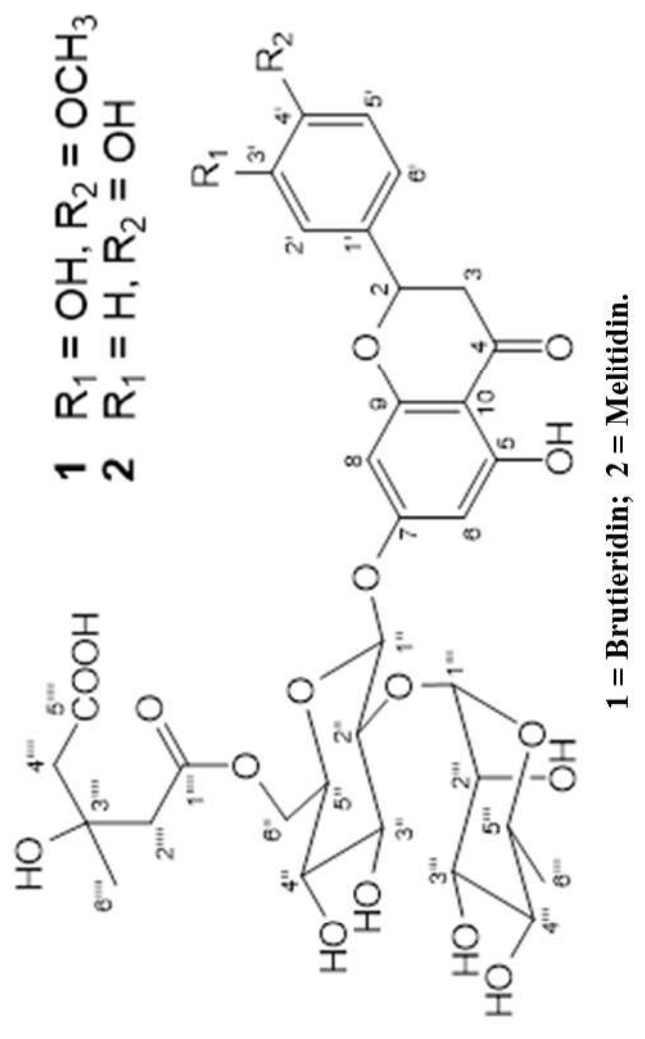

$\boldsymbol{m}$

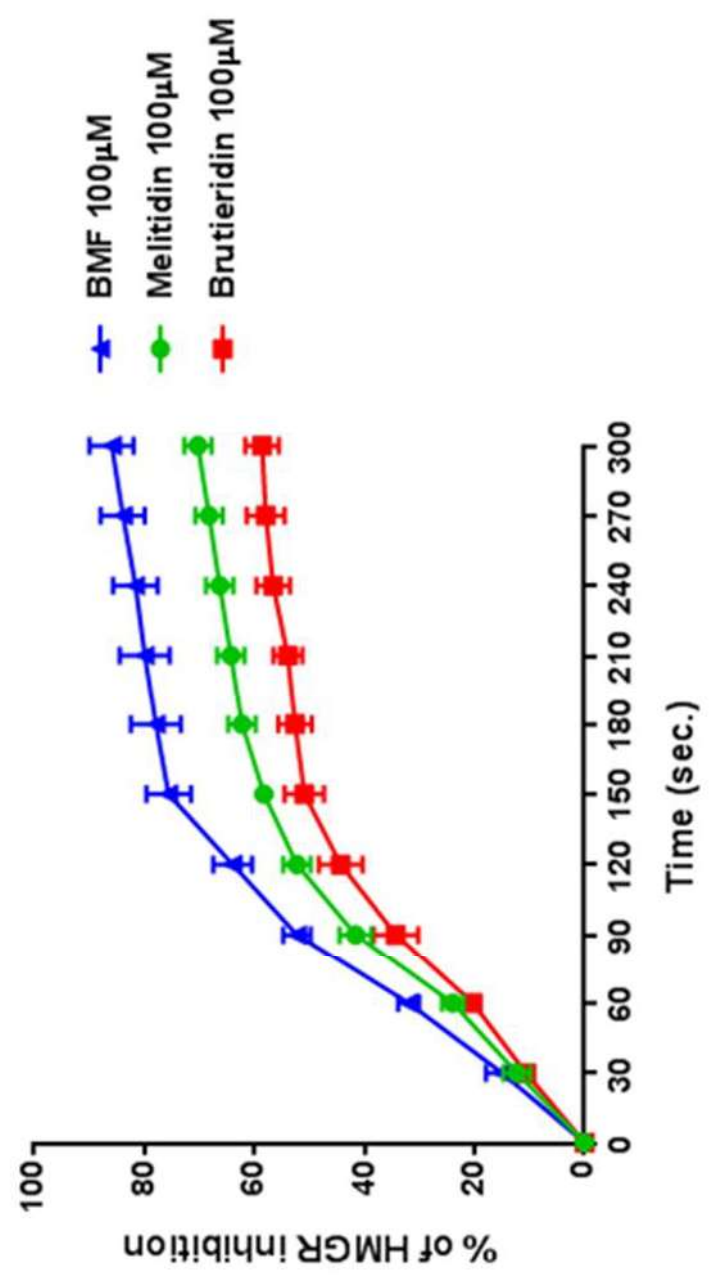

口
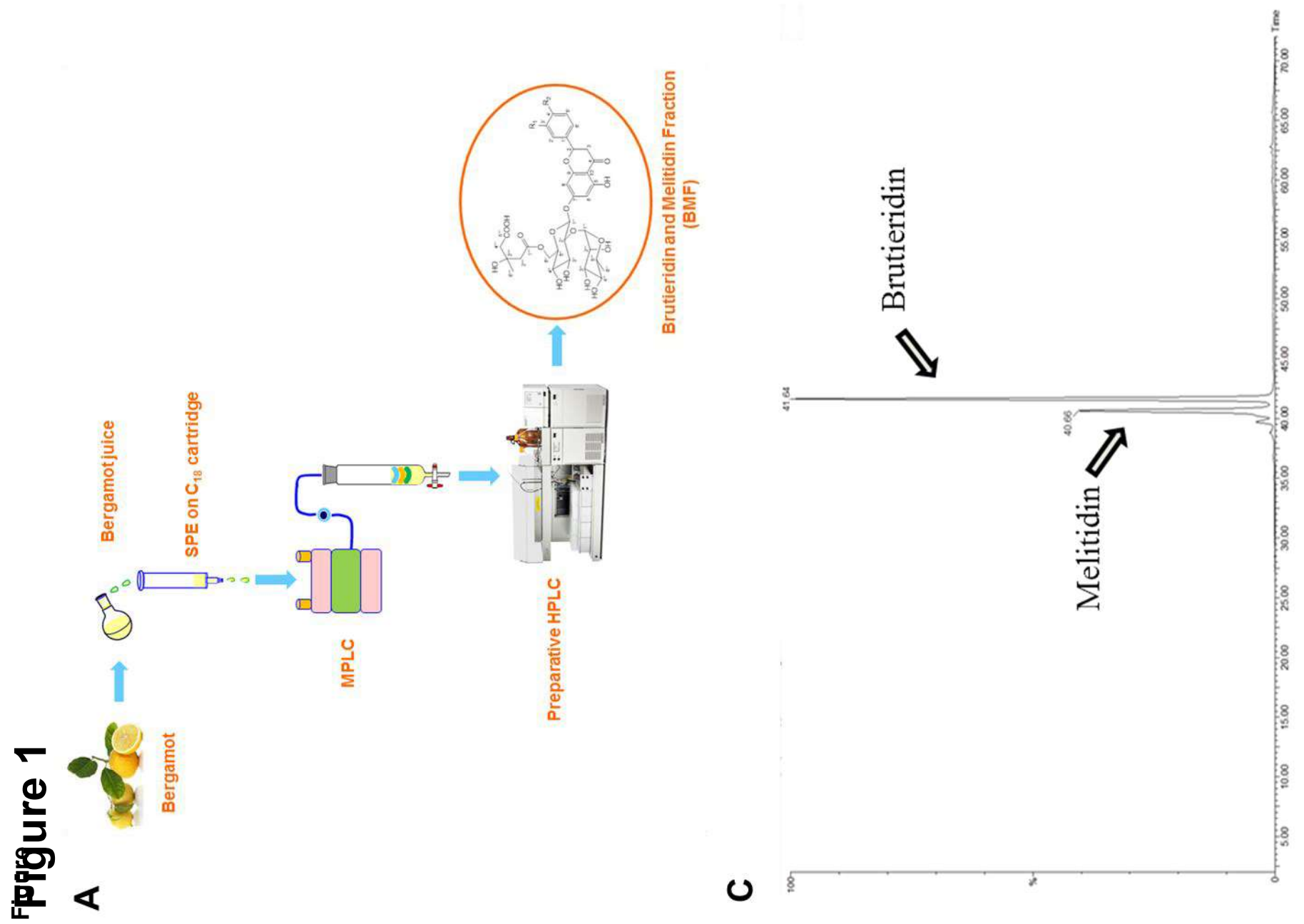

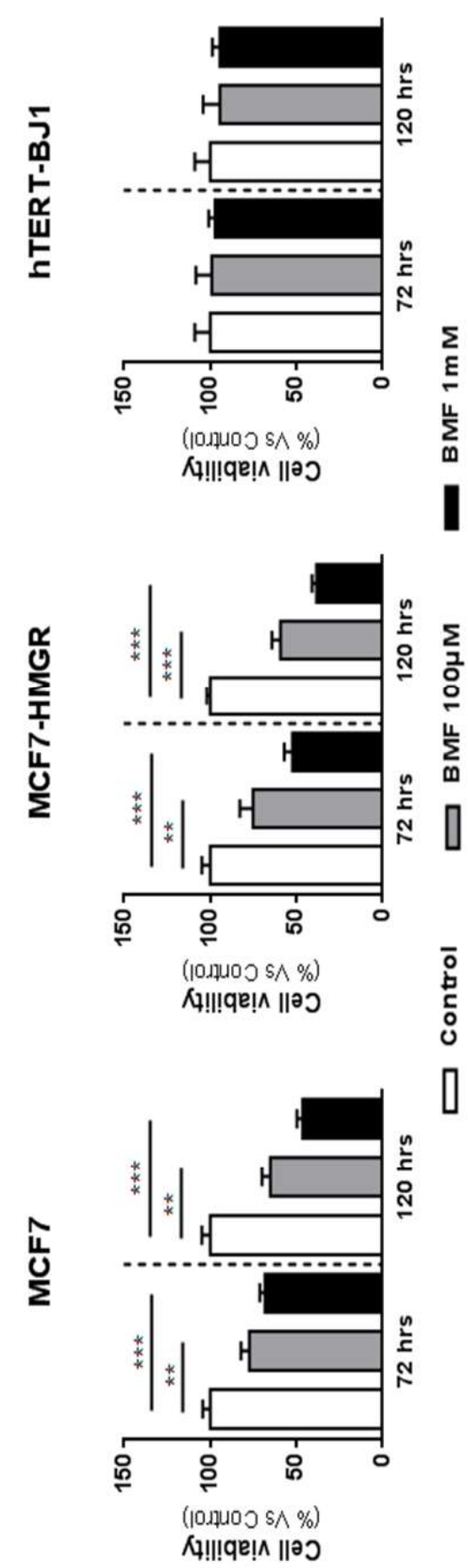

ต

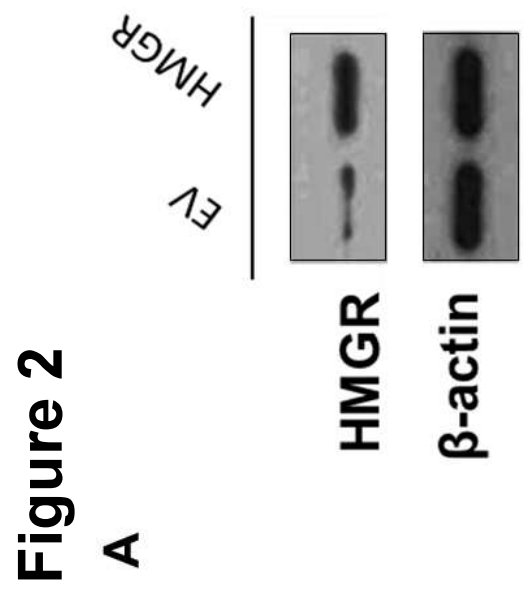

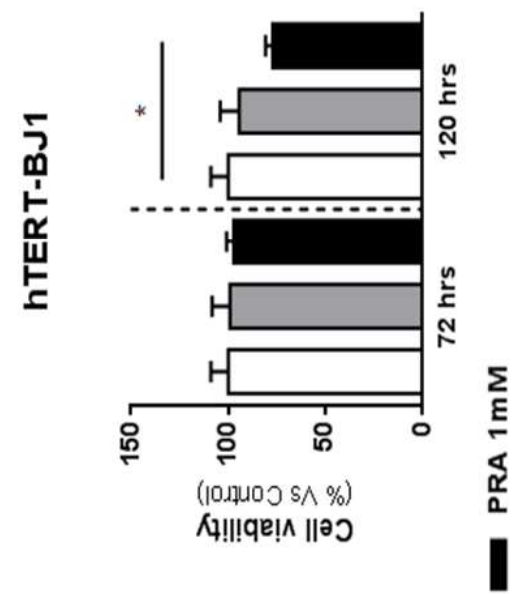
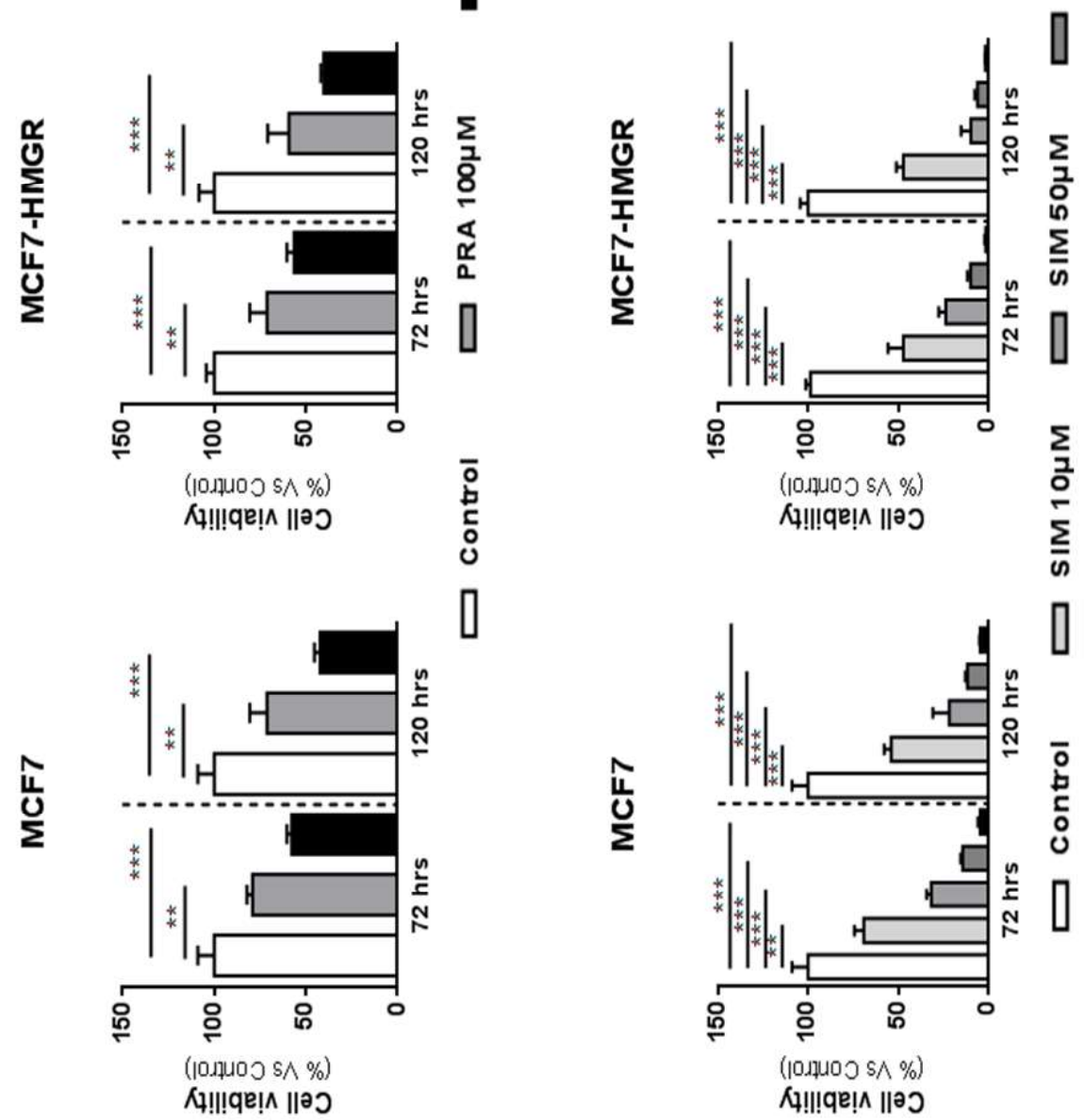

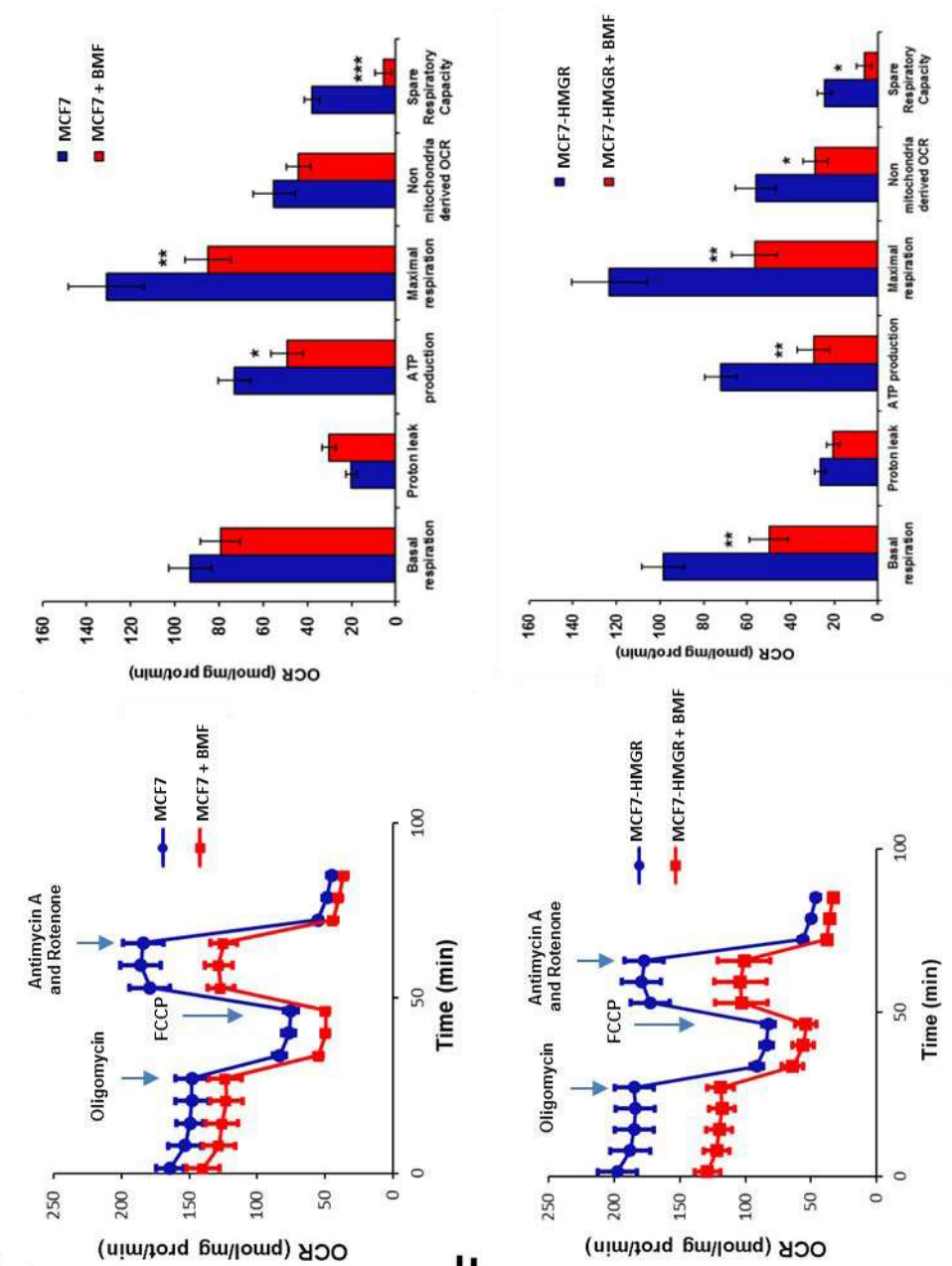

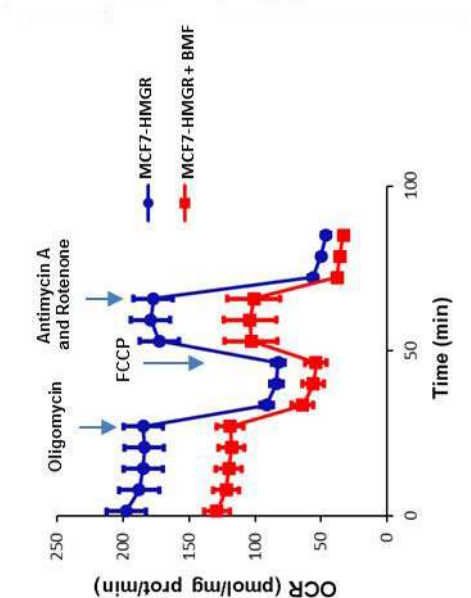

ш

ᄂ

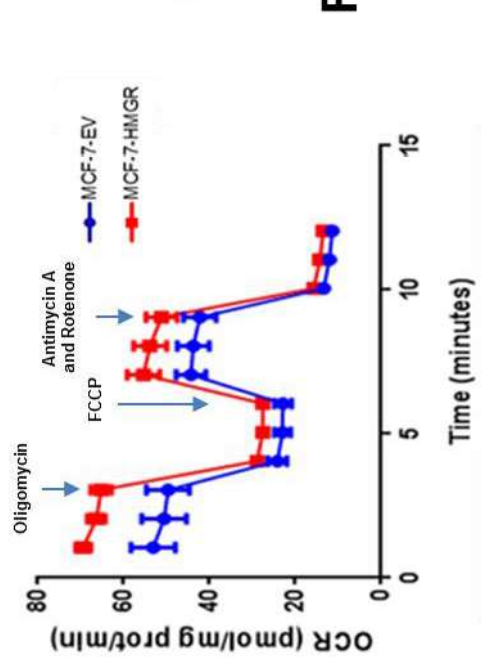

$m$

m

i

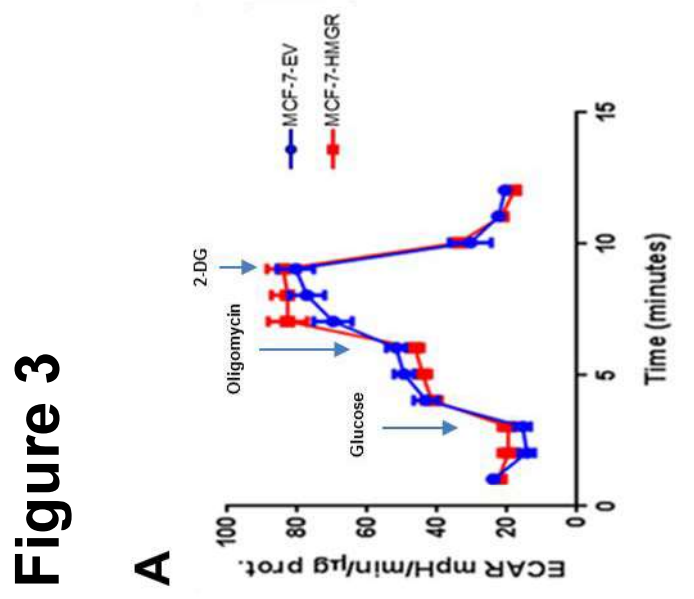

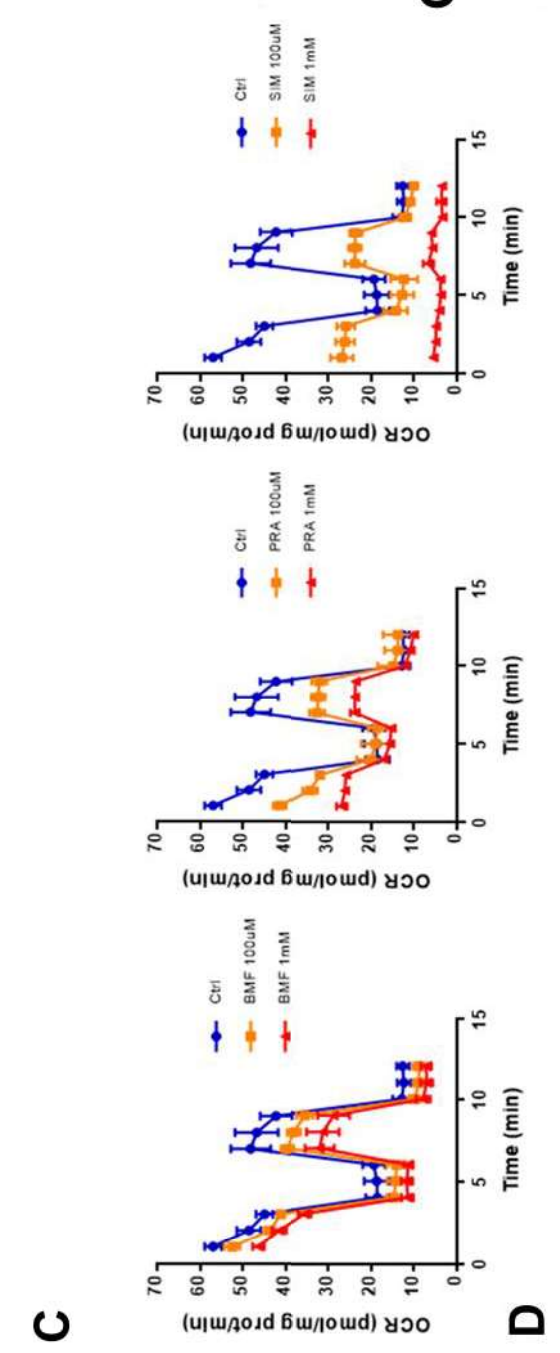

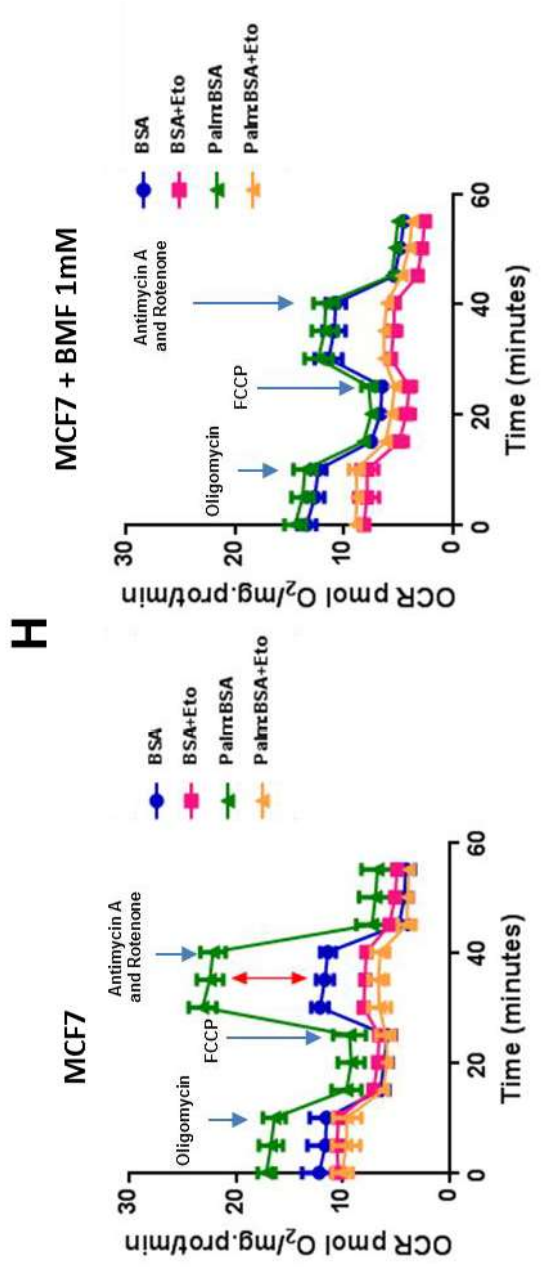

0

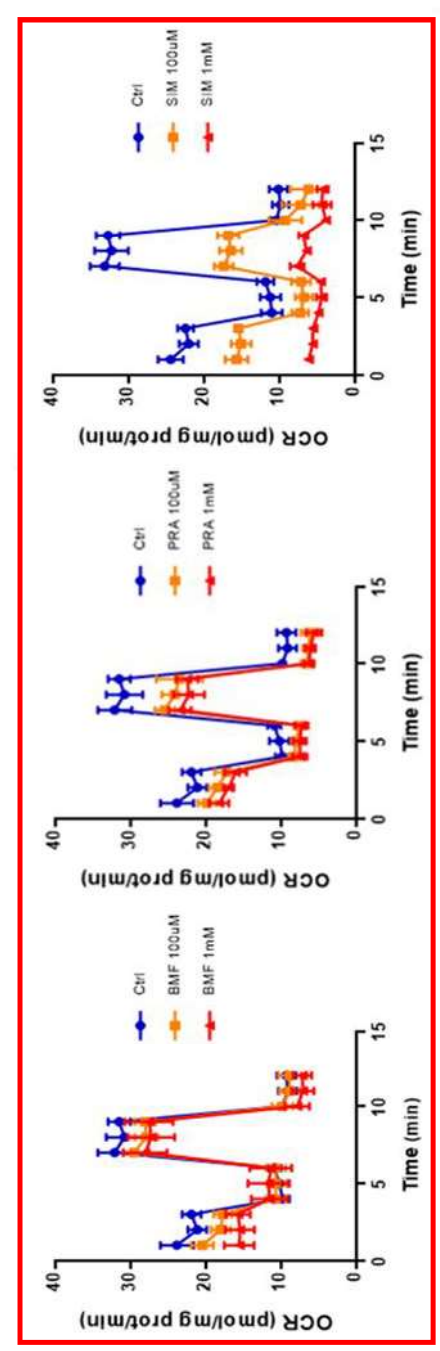




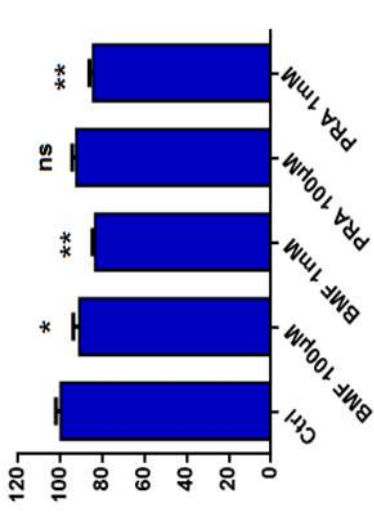

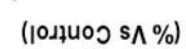

ן

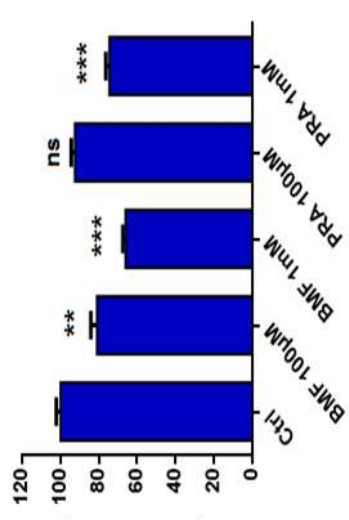

(10ג1uoo s^ \%) |w/.ม6d 8-7|

m
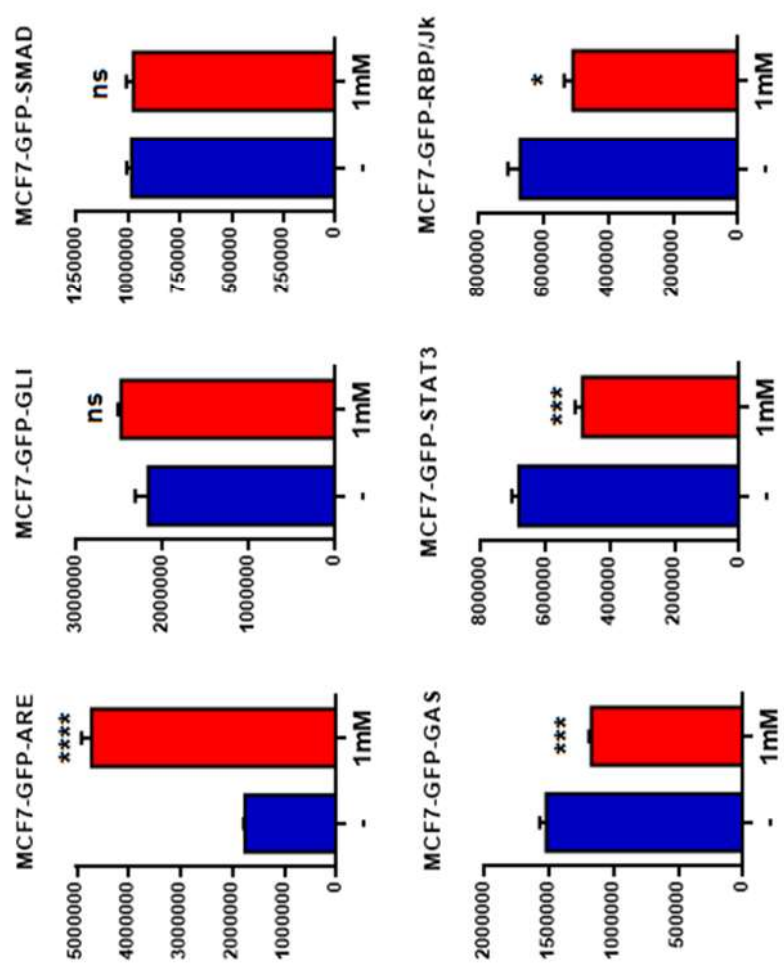

8

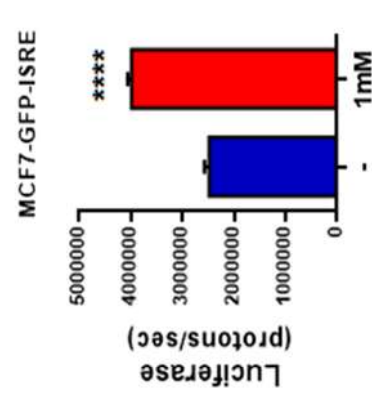

ir 4

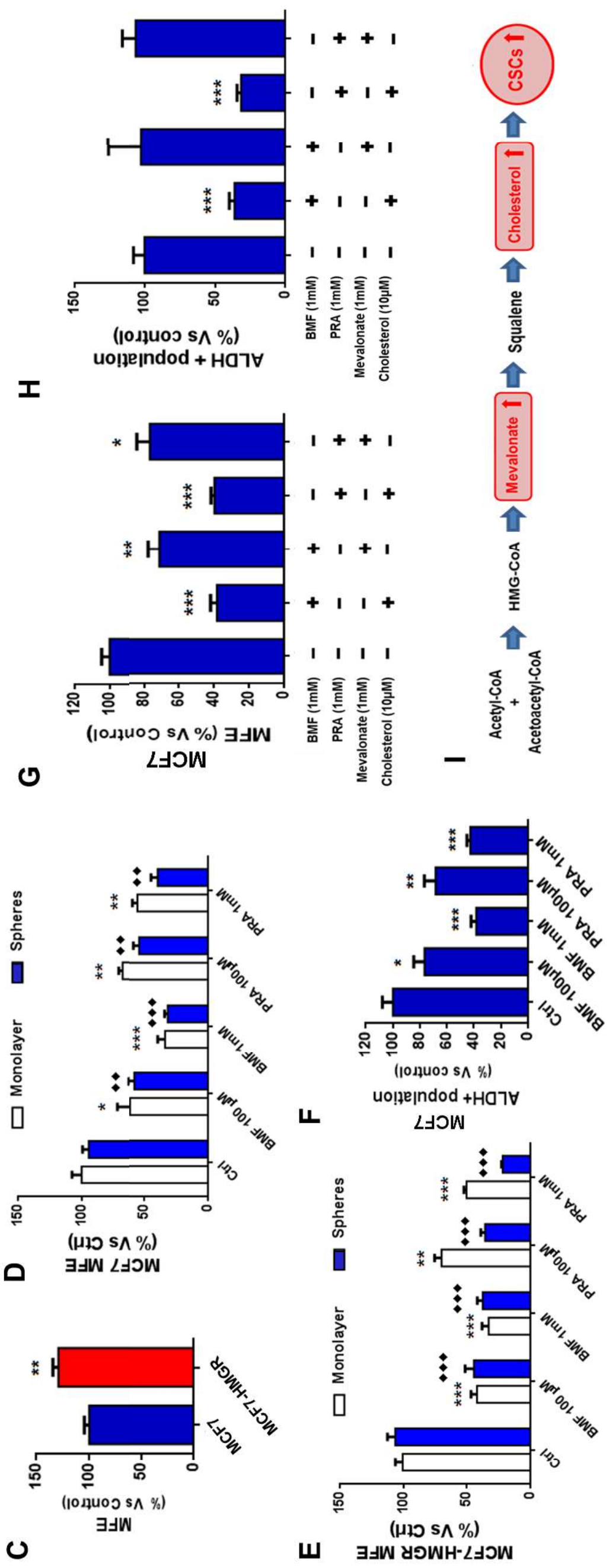

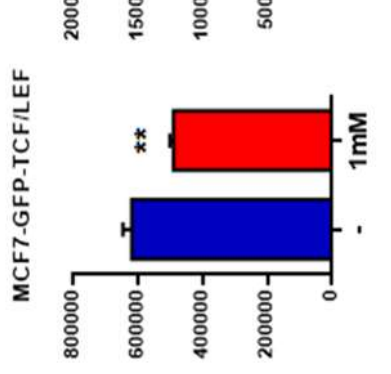



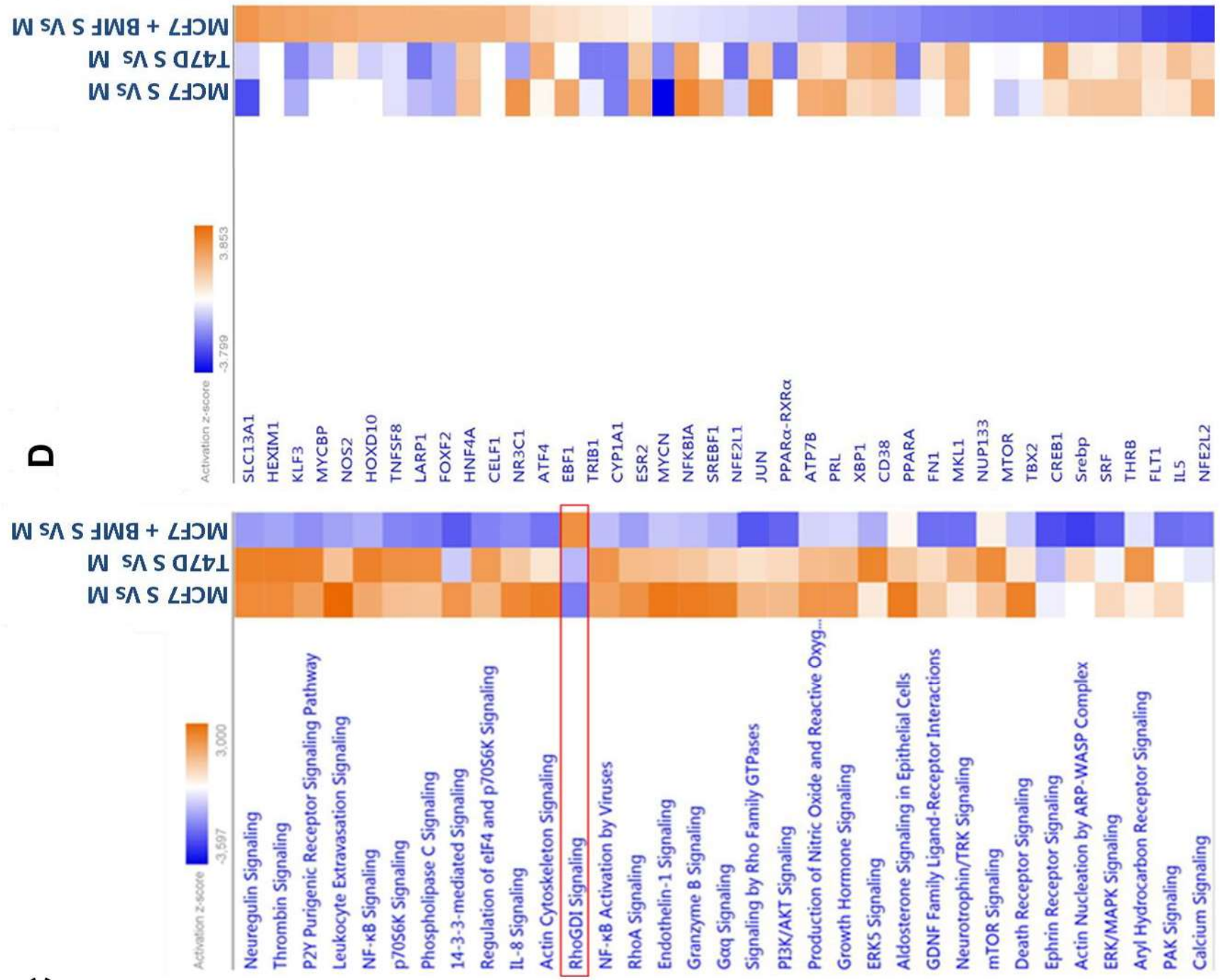

0
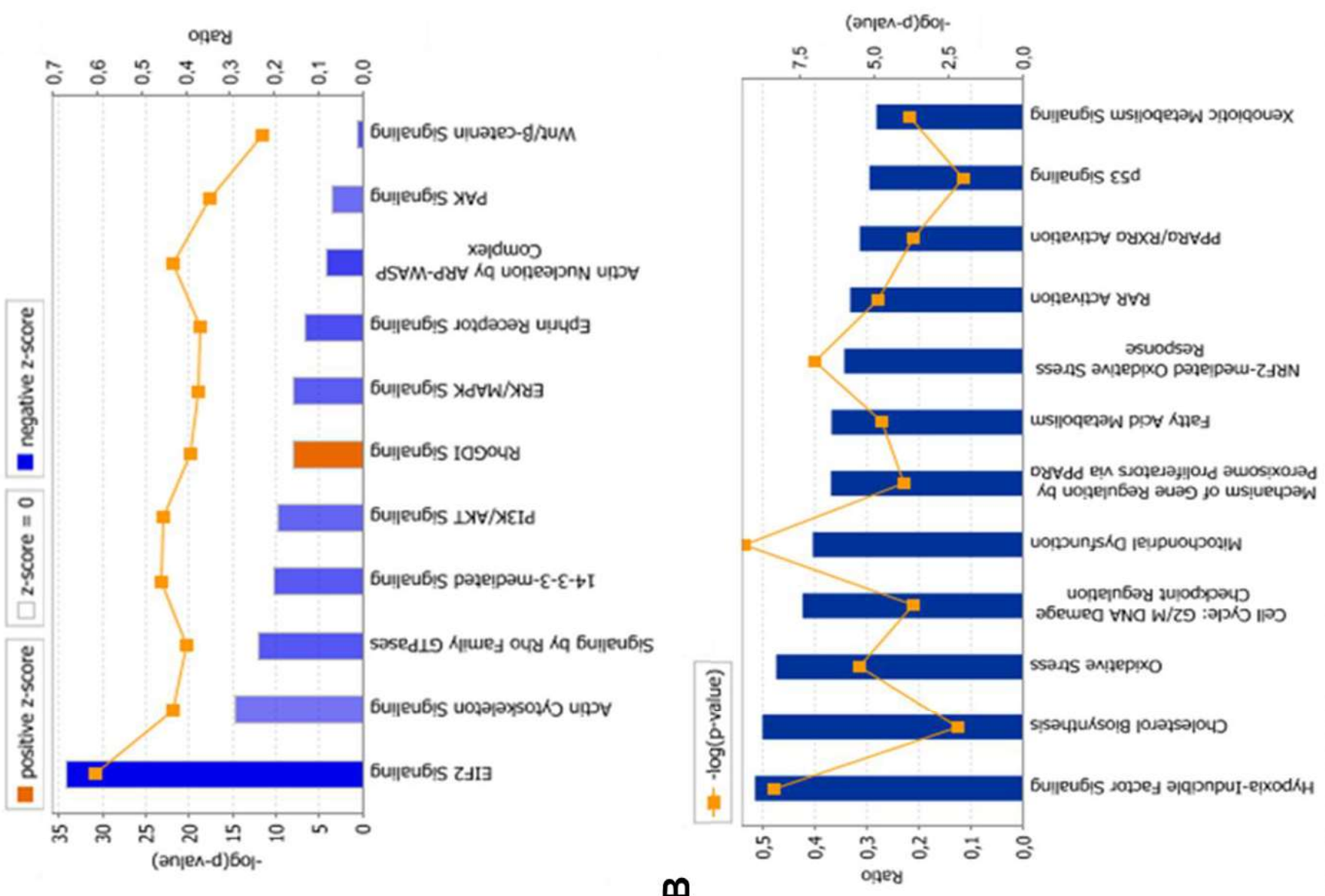

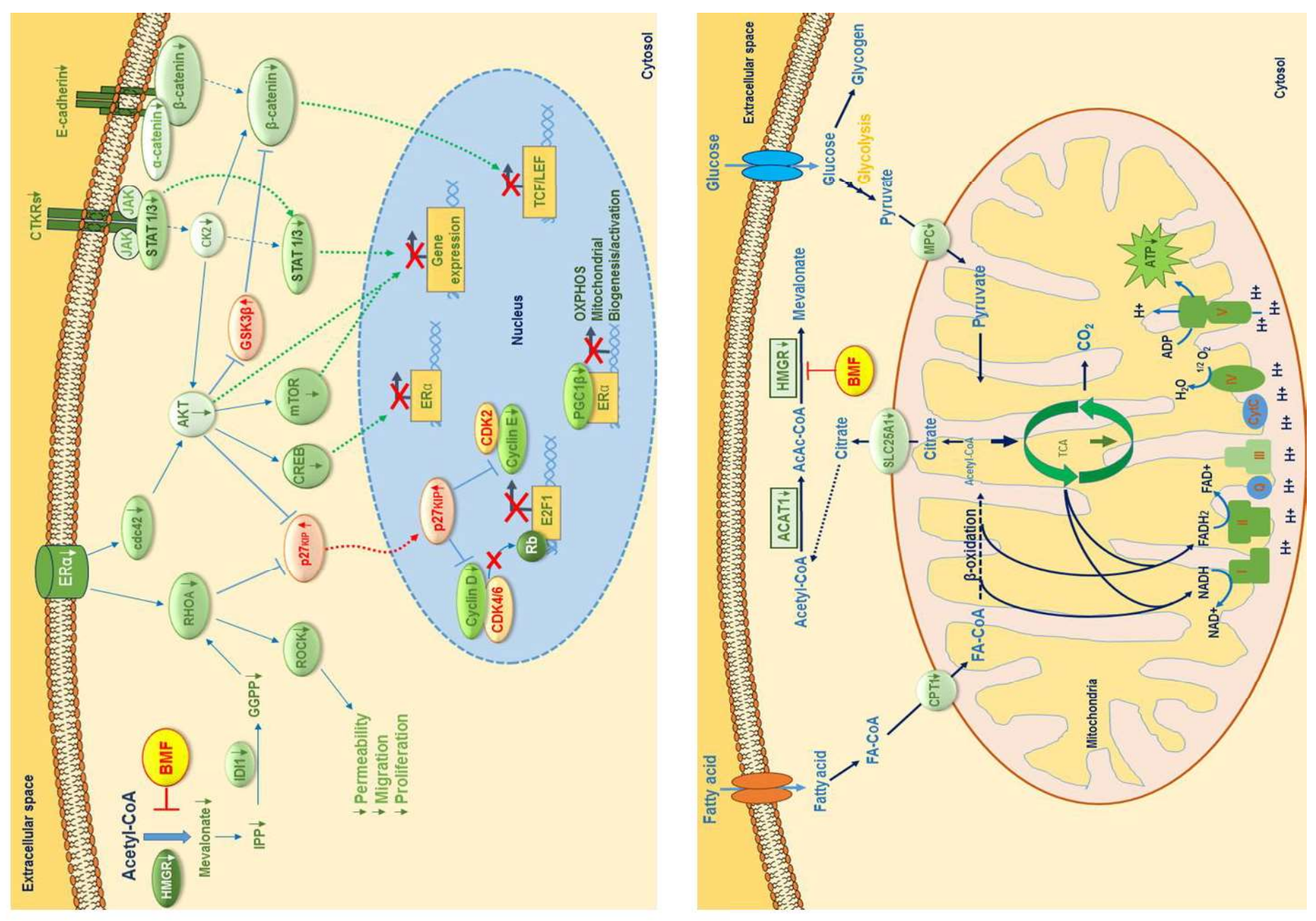

0

m

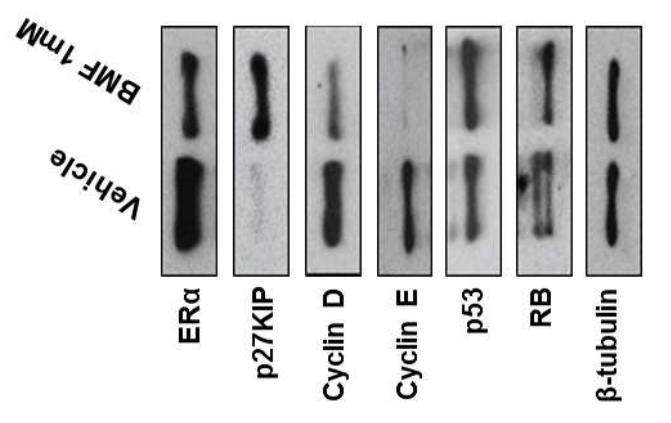

ᄂ

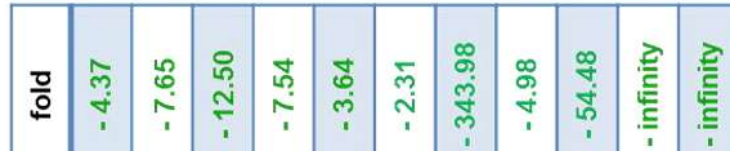

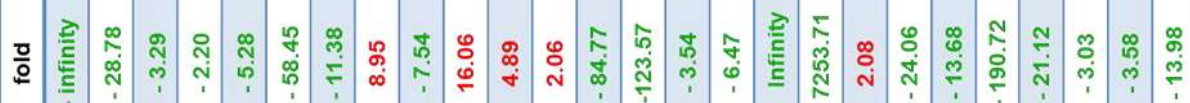
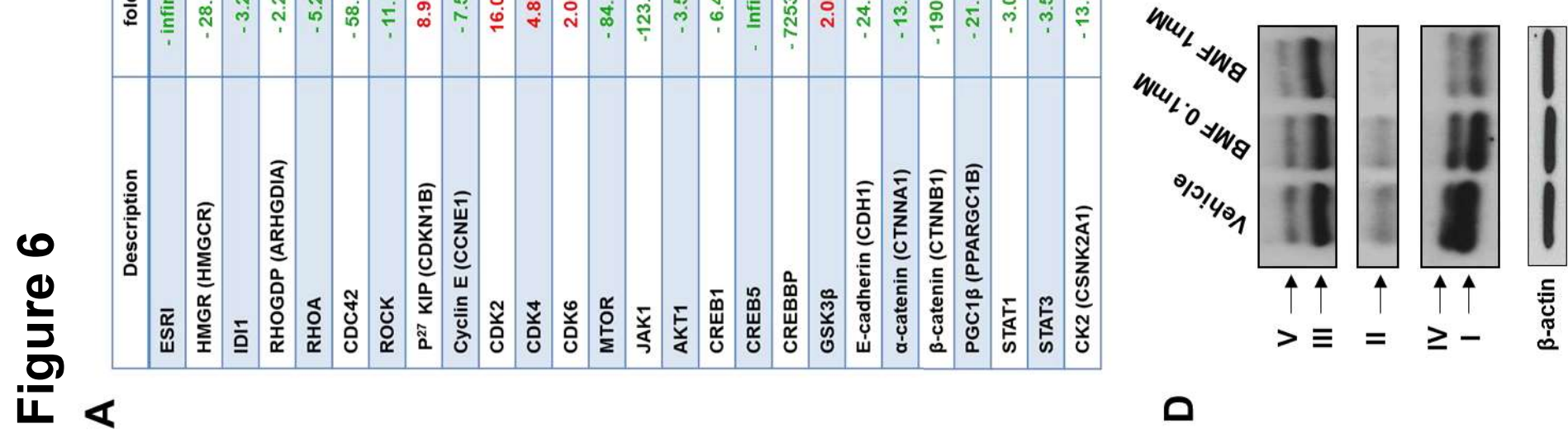

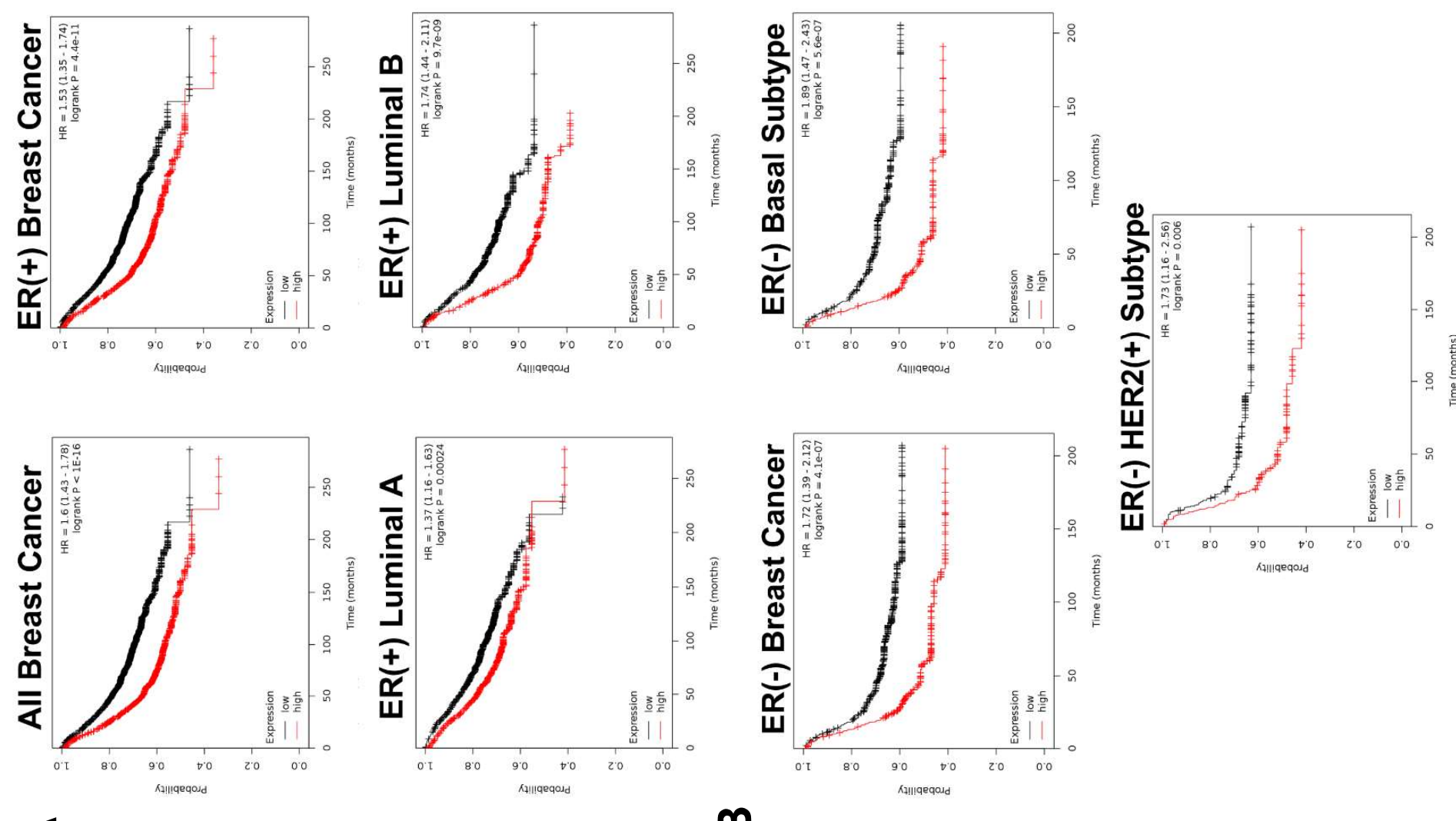

$\varangle$

m

온 


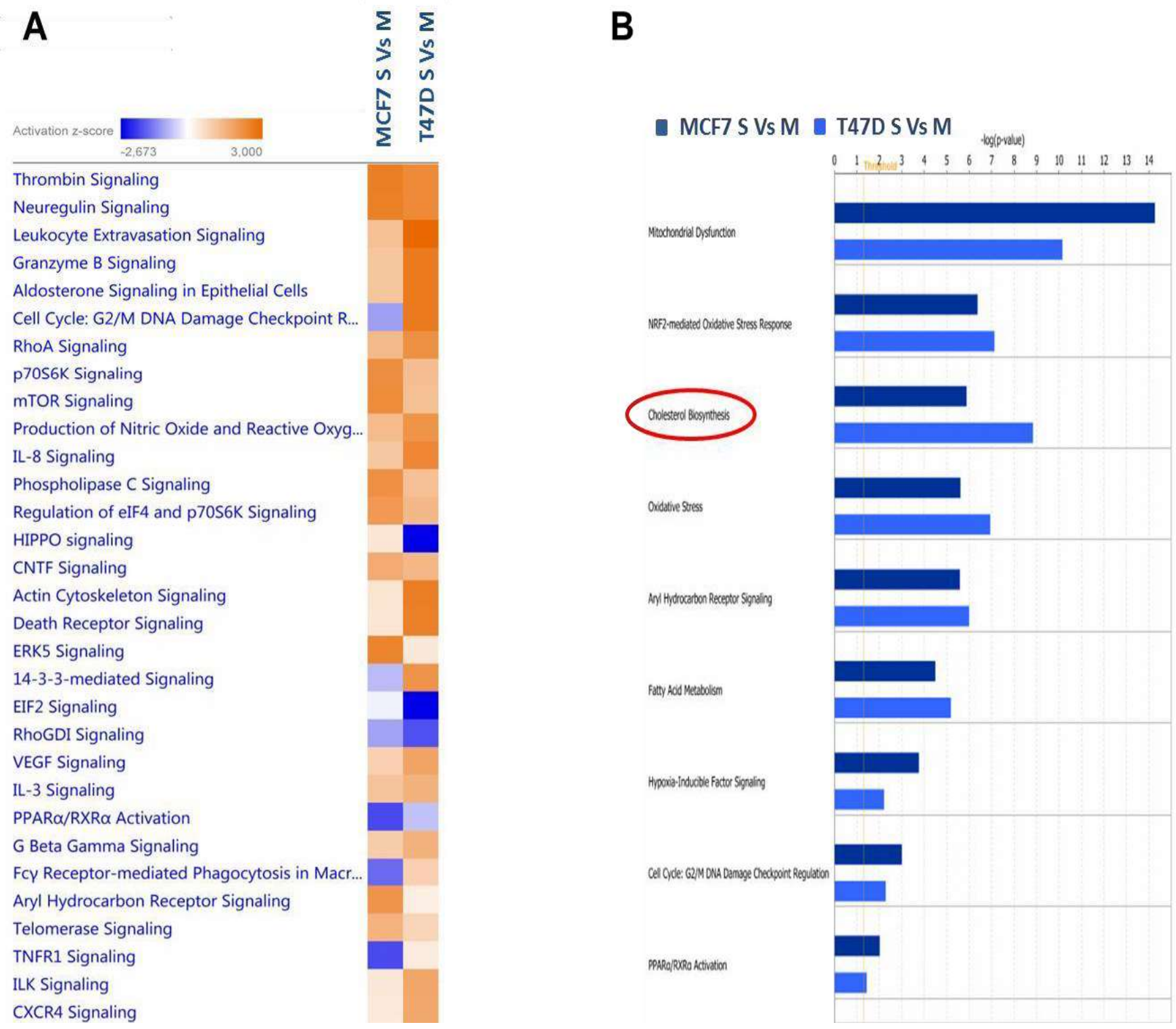

Figure S1: Ingenuity Pathway Analysis (IPA) of proteomics data sets obtained from human breast cancer cells, grown as either as 3D-spheroids (S) or cell monolayers (M). (A) Canonical pathways predicted to be altered in MCF7 and T47D 3D-spheroids (indicated with $S$ ), relative to control monolayer cells (indicated with an $\mathrm{M}$ ). A positive $\mathrm{z}$-score (orange color) represents the up-regulation of a specific pathway, while a negative z-score (blue color) indicates the down-regulation of a pathway.

(B) Toxicity effects of differentially expressed proteins in MCF7 and T47D 3D-spheroids (S), relative to control monolayer cells (M), are shown. Ingenuity Pathway Analysis showed that certain toxicity functions are significantly enriched by the proteins differentially expressed in this comparative analysis $(p<0.05)$. In the Bar chart, the $p$-value for each pathway is indicated by the bar and is expressed as -1 times the log of the p-value (cutoff $z$-score \pm 2 ). 
Mevalonate pathways-related proteins Upregulated in Mammospheres

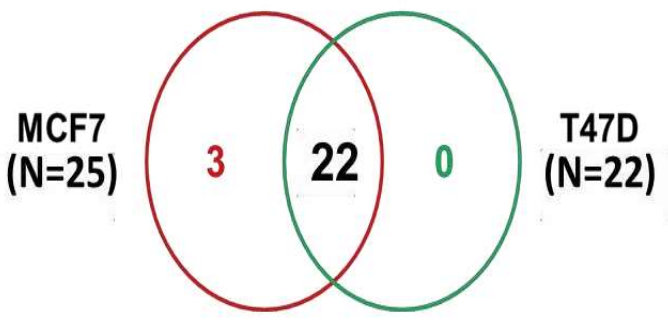

\begin{tabular}{|c|c|c|}
\hline MCF7 & Description & T47D \\
\hline Infinity & Isopentenyl-diphosphate Delta-isomerase 1 & Infinity \\
\hline Infinity & Ubiquitin carboxyl-terminal hydrolase & Infinity \\
\hline 3642,09 & Ubiquitin thioesterase & 14,06 \\
\hline 1124,93 & Acetyl-CoA acetyltransferase, mitochondrial & 49,45 \\
\hline 975,80 & NADH dehydrogenase [ubiquinone] 1 beta & 2,97 \\
\hline 522,86 & Delta(24)-sterol reductase & 30,01 \\
\hline 158,10 & Pyruvate carboxylase, mitochondrial & 2,59 \\
\hline 114,95 & Delta(3,5)-Delta(2,4)-dienoyl-CoA isomerase, mitochondrial & 10,49 \\
\hline 90,25 & Pyruvate dehydrogenase alpha 1 (PDHA1) & Infinity \\
\hline 38,50 & Lanosterol synthase (LSS) & 11,83 \\
\hline 20,60 & Squalene synthase & 7,00 \\
\hline 17,47 & NADH dehydrogenase [ubiquinone] 1 alpha & 6,47 \\
\hline 8,76 & 3-hydroxyl-3-methylglutaryl-Coenzyme A reductase & 6,64 \\
\hline 6,77 & Fatty acid synthase (FASN) & 8,53 \\
\hline 6,37 & 3'-hydroxyisobutyrate dehydrogenase & \#N/D \\
\hline 4,64 & Pyruvate kinase & 3,27 \\
\hline 3,57 & Carnitine 0 -acetyltransferase & 5,11 \\
\hline 2,59 & Isocitrate dehydrogenase [NADP] & 2,55 \\
\hline 2,41 & Malic enzyme & 2,45 \\
\hline 2,09 & Glyoxylate reductase/hydroxypyruvate reductase & 1,23 \\
\hline 1,93 & Mevalonate kinase & 2,10 \\
\hline 1,65 & Acetoacetyl-CoA synthetase & \#N/D \\
\hline 1,54 & 7-dehydrocholesterol reductase & \#N/D \\
\hline 1,49 & 3-hydroxy-3-methylglutaryl-Coenzyme A synthase 1 & 6,28 \\
\hline 1,25 & Diphosphomevalonate decarboxylase & 2,34 \\
\hline
\end{tabular}

Figure S2: Correlations of proteomics data sets in MCF7 and T47D

(A-B) Twenty-five proteins involved in the mevalonate pathway and cholesterol biosynthesis, were found to be up-regulated (fold change) in MCF7 mammospheres, as compared to MCF7 monolayer cells. Moreover, 22 proteins were found to be up-regulated in T47D mammospheres, as compared to T47D monolayer cells (all the proteins are listed in panel B). This represents an overlap of $88 \%$ (22 out of 25 ), as shown in the Venn diagram. 
Table S1. Prognostic Value of HMGR in Human Breast Cancer Sub-types:

Tumor Recurrences (RFS).

\begin{tabular}{|c|c|c|c|}
\hline Symbol & Gene Probe & HR (Hazard Ratio) & P-value (Log Rank Test) \\
\hline \multicolumn{4}{|c|}{ All Breast Cancers; $N=3,951$} \\
\hline HMGR/HMGCR & 202539_s_at & 1.60 & $<1 e-16$ \\
\hline HMGR/HMGCR & 202540_s_at & 1.38 & $6.9 e-07$ \\
\hline \multicolumn{4}{|l|}{$E R(+) ; N=3,082$} \\
\hline HMGR/HMGCR & 202539_s_at & 1.53 & $4.4 \mathrm{e}-11$ \\
\hline HMGR/HMGCR & 202540_s_at & 1.30 & $8.9 e-05$ \\
\hline \multicolumn{4}{|c|}{ ER(+)/Luminal $A ; N=1,933$} \\
\hline HMGR/HMGCR & 202539_s_at & 1.37 & 0.00024 \\
\hline HMGR/HMGCR & 202540_s_at & 1.26 & 0.0072 \\
\hline \multicolumn{4}{|c|}{ ER(+)/Luminal $B ; N=1,149$} \\
\hline HMGR/HMGCR & 202539_s_at & 1.74 & $9.7 e-09$ \\
\hline HMGR/HMGCR & 202540_s_at & 1.66 & $3.6 e-05$ \\
\hline \multicolumn{4}{|l|}{ ER(-); N=869 } \\
\hline HMGR/HMGCR & 202539_s_at & 1.72 & $4.1 \mathrm{e}-07$ \\
\hline HMGR/HMGCR & 202540_s_at & 1.70 & $1.3 e-05$ \\
\hline \multicolumn{4}{|c|}{ ER(-)/Basal; $N=618$} \\
\hline HMGR/HMGCR & 202539_s_at & 1.89 & $5.6 e-07$ \\
\hline HMGR/HMGCR & 202540_s_at & 1.71 & $9.1 \mathrm{e}-05$ \\
\hline \multicolumn{4}{|c|}{ ER(-)HER2(+); N=251 } \\
\hline HMGR/HMGCR & 202539_s_at & 1.73 & 0.006 \\
\hline HMGR/HMGCR & 202540_s_at & 1.60 & 0.032 \\
\hline
\end{tabular}


Table S2. HMGR Predicts Recurrence, Metastasis and Overall Survival in Breast Cancer Patients.

\begin{tabular}{|c|c|c|c|}
\hline Symbol & Gene Probe & HR (Hazard Ratio) & P-value (Log Rank Test) \\
\hline \multicolumn{4}{|c|}{$\begin{array}{l}\text { Tumor Recurrence (RFS); } \\
\text { All Breast Cancers; } \mathrm{N}=3,951\end{array}$} \\
\hline HMGR/HMGCR & 202539_s_at & 1.60 & $<1 \mathrm{e}-16$ \\
\hline HMGR/HMGCR & 202540_s_at & 1.38 & $6.9 e-07$ \\
\hline \multicolumn{4}{|c|}{$\begin{array}{l}\text { Distant Metastasis (DMFS); } \\
\text { All Breast Cancers; } N=1,746\end{array}$} \\
\hline HMGR/HMGCR & 202539_s_at & 1.71 & $4.3 e-08$ \\
\hline HMGR/HMGCR & 202540_s_at & 1.42 & 0.0017 \\
\hline \multicolumn{4}{|c|}{$\begin{array}{l}\text { Overall Survival (OS); } \\
\text { All Breast Cancers; } N=1,402\end{array}$} \\
\hline HMGR/HMGCR & 202539_s_at & 1.71 & $7.5 e-07$ \\
\hline HMGR/HMGCR & 202540_s_at & 1.35 & 0.0071 \\
\hline
\end{tabular}

